\title{
A mouse model of recurrent myocardial infarction reports diminished emergency hematopoiesis and cardiac inflammation
}

Sebastian Cremer1,2, Maximilian J. Schloss ${ }^{1,2}$, Claudio Vinegoni ${ }^{1,2}$, Shuang Zhang ${ }^{1,2}$, David Rohde $^{1,2}$, Paolo Fumene Feruglio ${ }^{1-3}$, Stephen Schmidt1,2, Greg Wojtkiewicz ${ }^{1,2}$, Ralph Weissleder ${ }^{1,2}$, Filip K. Swirski1,2, Matthias Nahrendorf1,2,4

${ }^{1}$ Center for Systems Biology, Massachusetts General Hospital Research Institute and Harvard Medical School, Boston, MA, USA.

2Department of Radiology, Massachusetts General Hospital, Boston, MA, USA.

3Department of Neurological, Neuropsychological, Morphological and Movement Sciences, University of Verona, Verona, Italy.

${ }^{4}$ Cardiovascular Research Center, Massachusetts General Hospital and Harvard Medical School, Boston, MA, USA.

Corresponding author: Matthias Nahrendorf Center for Systems Biology MGH Research Institute 185 Cambridge Street Boston, MA 02114 USA Tel: (617) 643-0500 Fax: (617) 643-6133 


\begin{abstract}
Recurrent $\mathrm{MI}$ is common in patients with coronary artery disease and associates with high mortality. Here we developed a surgical mouse model in which two subsequent Mls affect different left ventricular regions in the same mouse. Recurrent MI was induced by ligating the left circumflex followed by the left anterior descending branch of the coronary artery. We characterized the resulting ischemia by whole-heart fluorescent coronary angiography after optical organ clearing and by cardiac MRI. We report that a first MI induces bone marrow "memory" via a circulating signal, thereby affecting hematopoietic factor expression in bone marrow macrophages. This altered the organism's reaction to subsequent events. Inspite at least similar extent of injury reported by blood troponin, recurrent MI caused reduced emergency hematopoiesis and less leukocytosis than a first MI. Consequently, fewer leukocytes migrated to the ischemic myocardium. The hematopoietic response to lipopolysaccharide was also mitigated after a previous MI. Our data suggest that hematopoietic and innate immune responses are shaped by a preceding MI.
\end{abstract}




\section{Introduction}

Recurrent myocardial infarction (MI) is a common clinical problem and an independent predictor of morbidity and mortality in patients (Thune et al., 2011; Stone et al., 2014). Even with contemporary primary percutaneous intervention, reinfarction still occurs in 1 of 10 patients. One-year mortality in patients with recurrent $\mathrm{Ml}$ is about four times higher than in patients with a first infarct $(38.3 \%$ versus $10.3 \%)$, and $20 \%$ of patients with recurrent $\mathrm{MI}$ die within seven days(Thune et al., 2011). Mortality is at its peak within the first month after the index $\mathrm{MI}$ (Thune et al., 2011). The poor outcome after recurrent $\mathrm{MI}$ is attributed to additional loss of viable myocardium and development of heart failure, but mechanistic studies investigating the underlying causes are lacking.

Myocardial infarction is a sterile injury that elicits an immune response to replace necrotic cardiac cells with a robust scar (Nahrendorf, 2018). A hallmark of this reaction is massive recruitment of short-lived neutrophils and monocytes from hematopoietic organs, which increase myeloid cell production by a process called emergency hematopoiesis (Nahrendorf et al., 2007; Swirski et al., 2009; Dutta et al., 2015b). Newly made leukocytes enter the heart and deploy a variety of molecular cues, namely cytokines, proteases and angiogenic substances, to enable myocardial healing. A sufficient inflammatory reaction is necessary for optimal healing after $\mathrm{Ml}$; however, unrestrained myeloid cell activity results in adverse cardiac remodeling and heart failure (Frangogiannis, 2012).

Recently the concept of "trained immunity" (Netea et al., 2016) reinvigorated interest in changes to innate immune cells' functional programs. After inflammatory stimuli, i.e. "training", more vigorous activity occurs in response to subsequent events, while states of tolerance occur after lipopolysaccharide challenge (Foster et al., 2007; Saeed et al., 2014). The initial findings obtained in macrophages have now been extended to bone marrow hematopoiesis and "training" with IL-1 $\beta$ and atherogenic diet (Christ et al., 2018; Mitroulis et al., 2018). Conceptually, this body of work indicates that the innate immune system may react non-uniformly depending on prior challenges to the equilibrium. Within this framework, the innate immune response to MI may also depend on prior stimuli, yet it is currently unclear whether a second infarct elicits a response identical to that observed after a first MI. Motivated by the importance of leukocyte recruitment after MI for acceleration of atherosclerosis and for heart failure development (Nahrendorf, 2018), we hypothesized that the inflammatory signals inducing emergency hematopoiesis after $\mathrm{Ml}$ also have consequences for recurrent myocardial ischemia.

Since there was no method available to test this hypothesis, we developed a model of recurrent $\mathrm{MI}$, which we describe for the first time in this Resource Article. In the mouse, the major part of the left ventricle is supplied by the left coronary artery, which emerges under the left atrium and continues to the apex of the heart (Fernández et al., 2008). It begins as a main trunk of 2-3mm length. Below the tip of the left atrial appendage, the artery divides into i) a branch with significant caliber that supplies blood to the posterolateral area of the heart (left circumflex, LCX)(Ahn et al., 2004; Fernández et al., 2008); ii) a branch that continues to the apex of the heart and provides blood to the anterior wall of the heart (left anterior descending, LAD)(Ahn et al., 2004; Fernández et al., 2008). We first ligated the LCX and later separately ligated the LAD branch to cause a second $\mathrm{MI}$ in the same mouse. We used whole heart fluorescence coronary angiography (FCA) and delayed gadolinium enhancement magnetic resonance imaging (MRI) to map the sequelae of recurrent MI. We then tested the hypothesis that recurrent MI induces an immune response that diverges from a first infarct. 


\section{Results \\ A mouse model of recurrent MI}

Investigating recurrent multifocal ischemia in mice requires a method that allows subsequent Mls at different locations in the same mouse heart. The left coronary artery's first branching point gives rise to the left anterior descending artery (LAD) and a circumflex artery (LCX) (Fernández et al., 2008). Since both arterial branches are fairly large caliber, we devised a model in which each is separately ligated distal of the branching point (Figure 1A, B). To explore both surgeries' outcome, we performed whole heart fluorescent coronary angiography (FCA) after optical tissue clearing (Richardson and Lichtman, 2015) and in vivo labeling of the cardiac vasculature with a fluorescent dye. Without tissue clearing, coronary arteries were not visible because they are partially covered by myocardial tissue, which efficiently absorbs and scatters photons (data not shown). We therefore adapted CUBIC (clear, unobstructed brain imaging cocktails and computational analysis), a tissue clearing method previously developed for brain imaging (Susaki et al., 2014). Tissue clearing was done after injection of fluorescein-labelled albumin, which served as a fluorescent vascular imaging agent (see method section). FCA clearly visualized the coronary arteries and their branches LAD and LCX (Figure 1C). Ligating the LCX branch induced a strictly posterolateral infarct that did not extend to the apex of the heart (Figure 1D and Figure S1), while the LAD ligation distal of the main branching point affected a large portion of the anterior wall including the apex (Figure 1E). Finally, after both ligations, a very large infarct covering a major area of the left ventricle emerged (Figure 1F, Figure S1).

To examine the consequences of these surgeries, we conducted a cardiac MRI study with delayed gadolinium enhancement, a gold standard for identifying non-viable myocardium in mice and humans (Kim et al., 2000; Yang et al., 2004) (for timeline, see Figure 1G). LCX ligation created posterolateral, gadolinium-enhanced infarcts that significantly reduced the ejection fraction two days after Ml, while the dimensions of the left ventricle remained unchanged at this early time point after ischemia (Figure $1 \mathrm{H}, \mathrm{I}$ and supplementary video 1). Additionally infarcting the $L A D$ in the same mouse two days later generated larger infarcts, reduced the left ventricular ejection fraction and induced dilation of the left ventricle (Figure $1 \mathrm{H}, \mathrm{I}$ and supplementary video 2). Twenty-one days after the first MI (17 days after the second), the left ventricle dilated further (Figure 1I).

\section{Hematopoietic response to a single LAD versus a single LCX infarct}

Appropriate leukocyte supply is necessary to promote optimal healing of the infarcted heart, as recruited immune cells remove dead myocytes and orchestrate wound healing (Swirski et al., 2009). Most recruited cells are innate immune cells, with short half lives, that are supplied by hematopoietic tissues. Ml activates bone marrow to generate a sufficient leukocyte supply to the heart in mice and humans(Dutta et al., 2015b). We first tested whether lateral infarcts after LCX ligation induce a bone marrow activation similarly to previously described LAD infarcts (Dutta et al., 2015b). Comparing lineage- ckit $^{+}$sca-1+ (LSK) progenitor cells and most upstream CD150+ CD48+ hematopoietic stem cell (SLAM HSC) numbers in the mice femurs after LCX ligation versus LAD ligation or naive controls, we found that HSPC numbers increased after LCX ligation when compared to naive controls, albeit not to the same extent as after LAD ligation (Figure 2A, B), likely because LCX infarcts are smaller than LAD infarcts. The number of downstream granulocyte macrophage progenitors (GMP) increased robustly after either LCX or LAD ligation (Fig. 2B).

\section{Recurrent MI triggers attenuated systemic inflammation}

It was unclear whether prior MI alters the hematopoietic emergency response to a second infarct. To investigate, we designed an experiment that compares the inflammatory response after permanent LAD ligation in mice that either had or had not received a LCX ligation 10 days before (Figure 3A). We first evaluated whether emergency hematopoiesis had returned to steady state 10 days after MI (Figure S2A). Indeed, at this time point, the bone marrow's hematopoietic stem and progenitor cell (HSPC) numbers, which expand significantly in the days after MI (Dutta et al., 2015b), had dropped to normal levels (Figure 
S2B, C). Likewise, neutrophil and monocyte numbers were comparable in naive mice and mice 10 days after LAD ligation (Figure S2D). We concluded that a 10-day interval between the LCX and LAD ligation is appropriate because $\mathrm{i}$ ) at that point hematopoietic cell numbers have returned to steady state, ii) the time interval is clinically relevant (Thune et al., 2011; Stone et al., 2014) and iii) a comparable time interval has previously been used to study "trained immunity" (Saeed et al., 2014; Mitroulis et al., 2018). We thus settled on an experimental design in which a control cohort receives only LAD ligation, while the experimental cohort receives an LAD ligation 10 days after a preceding LCX infarct (recurrent MI, Figure 3A). Blood troponin levels indicated that the acute myocardial damage caused by permanent LAD ligation was not smaller in mice with prior LCX ligation (Figure S3).

We next compared innate immune cell numbers 3 days after a first MI (LAD only) to 3 days after recurrent MI (LAD with preceding LCX). Surprisingly, blood monocyte and neutrophil numbers were lower in mice with recurrent $\mathrm{Ml}$ (Figure 3B, C). This was accompanied by lower levels of neutrophils and monocytes in the bone marrow (Figure 3D, E), indicating leukocyte production - rather than only release - is reduced in the marrow. Indeed, LSK and GMP numbers were significantly lower on day 3 after recurrent MI (Figure 3F, G), when post-MI emergency hematopoiesis peaks (Dutta et al., 2015b). In alignment with these HSPC numbers, LSK and GMP proliferation was lower in the femurs of mice with recurrent $\mathrm{MI}$ than after a first infarct, as shown by analyzing cell proliferation using a BrdU incorporation assay (Figure $3 \mathbf{H}, \mathbf{I})$. This trend did not reach statistical significance for upstream SLAM HSC (Figure $\mathbf{3 H}, \mathbf{I}$ ).

\section{Recurrent MI changes the hematopoietic niche}

In addition to growth factors and danger signals, HSPC proliferation and leukocyte production is governed by the hematopoietic niche, specifically by proteins encoded by the genes cxcl12, angpt1, kitl and vcam1 (Mendelson and Frenette, 2014; Morrison and Scadden, 2014). Expression of these genes was significantly higher in mice with recurrent $\mathrm{MI}$ compared to those with a first infarct, except for angpt1 (Figure 3J). These data implicate the involvement of the bone marrow niche, which instructs HSPC activity, in our observation of lower hematopoietic expansion after recurrent MI.

\section{Preceding ischemia reperfusion injury restricts post-MI emergency hematopoiesis}

Next we extended the above findings to ischemia/reperfusion injury (IRI). We instigated ischemia by blocking LAD branch blood supply for 30 minutes, followed by reperfusion of the occluded vessel. Ten days later mice underwent permanent ligation of the LAD while controls received LAD ligation without preceding IRI (Figure 4A). Troponin levels in mice in which IRI preceded MI were not below those of control mice that only underwent LAD ligation (Figure 4B). Similar to the observations in recurrent MI described above, mice with prior IRI had lower bone marrow LSK and GMP numbers three days after permanent LAD ligation (Figure 4C, D). Likewise, HSPC proliferation was significantly lower as assessed by BrdU incorporation (Figure 4E, F). These data indicate that a "memory" of the bone marrow compartment can be induced not only by non-reperfused but also reperfused MI.

\section{A circulating factor dampens emergency hematopoiesis in recurrent $\mathrm{MI}$}

After myocardial ischemia, the bone marrow is activated by various mechanisms, such as direct signaling of sympathetic nerves (Dutta et al., 2012) or by soluble factors, which are produced in the injured myocardium (Sager et al., 2015). To test whether a circulating factor limits emergency hematopoiesis after recurrent $\mathrm{MI}$, mice were joined in parabiosis to create a shared circulation. After two weeks of parabiosis, the time necessary to create equilibrium between both mice, one parabiont received an LAD MI while the other parabiont did not. Five days later, mice were split and the non-infarcted parabiont was subsequently subjected to an LAD MI in both cohorts. Mice in the experimental group were therefore exposed to the blood of the infarcted parabiont 10 days prior to MI. Mice joined in parabiosis without prior $\mathrm{MI}$ were given LAD ligation and used as controls (Figure 5A). 
We found that LSK and GMP numbers were lower in the bone marrow of mice in which a parabiont had previous MI surgery (Figure 5B, C). Accordingly, we found lower proliferation rates of LT-HSC, MPP, LSK and GMP in the bone marrow of these mice (Figure 5D, E). This lower proliferation resulted in decreased numbers of myeloid cells in the blood (Figure 5F, G), which serves as a conduit for cells migrating to the infarct. Indeed, we detected fewer myeloid cells in the infarcted myocardium three days after $\mathrm{Ml}$ in mice in which the parabiont had experienced prior $\mathrm{MI}$ (Figure $\mathbf{5 H}, \mathbf{I})$. Taken together, these data demonstrate that a soluble signal travels from the infarcted parabiont to the non-infarcted parabiont's bone marrow, where it dampens bone marrow activation after a recurrent ischemic event. Hypothetically, IL-1 $\beta$ may have such a role, given that it alerts the marrow after MI (Sager et al., 2015) and that it has lasting effects on hematopoiesis in hyperlipidemia (Christ et al., 2018).

\section{Prior MI attenuates LPS-induced emergency hematopoiesis}

To test if these observations extend to non-ischemic recall challenges, we conducted experiments in which the bacterial wall component lipopolysaccharide (LPS) served as a secondary challenge ten days after MI (Figure 6A). This experimental design follows classical "trained immunity" experiments, which likewise rely on LPS recall (Saeed et al., 2014; Mitroulis et al., 2018). The bone marrow was analyzed 24 hours after injection of LPS, which induces severe systemic inflammation and emergency myelopoiesis to meet the organism's demand for myeloid cells to fight bacteria (Mitroulis et al., 2018). After LPS injection, we found fewer HSC and LSK as well as significantly reduced progenitor cell numbers in the bone marrow of mice with prior MI (Figure 6B-D). In addition, we observed a non-significant trend towards reduced neutrophil and monocyte numbers in the bone marrow of mice that underwent MI 10 days prior to LPS exposure (Figure 6E). As after recurrent MI, leukocyte production was diminished, indicated by lower BrdU incorporation in HSPC of mice with MI prior to LPS injection (Figure 6F, G). In sum, these data indicate that ischemic injury of the heart induces "tolerance" or exhaustion in the bone marrow, preventing robust HSPC expansion and generation of sufficient amounts of innate immune cells after reexposure to inflammatory stimuli.

\section{Macrophage ablation abolishes bone marrow "tolerance"}

Macrophages reside in the bone marrow and are a long-lived component of the hematopoietic niche (Chow et al., 2011; Chow et al., 2013), which governs HSPC quiescence, maintenance and proliferation. In the bone marrow, macrophages were characterized as CD3- B220- Gr1-CD115- F4/80+ SSC ${ }^{\circ}$. As previously described (Chow et al., 2011), we found macrophages to be MHCIlintermed Cx3cr1neg CD169high Vcam 1high (Figure S4). Given prior reports of trained immunity and tolerance (Foster et al., 2007; Biswas and Lopez-Collazo, 2009; Cheng et al., 2014; Saeed et al., 2014), we hypothesized that bone marrow macrophages may be involved in retaining "memory" of MI. To test this hypothesis, we depleted macrophages with clodronate liposomes in mice with or without prior MI (Figure 7A) and examined the effect of LPS on HSPC expansion. Clodronate efficiently depleted macrophages in the bone marrow (Figure 7B, C). We reproduced the attenuated myeloid cell expansion described above in mice with prior MI that received LPS and control liposomes compared to mice without MI (Figure 7D, E). After macrophage ablation, HSPC numbers fell even in the absence of MI (Figure 7D, E), which is in accordance with prior reports suggesting that niche macrophages maintain HSPC, for instance via the adhesion molecule Vcam-1 (Chow et al., 2011; Chow et al., 2013; Dutta et al., 2015a). Importantly, in the absence of macrophages, progenitor cell numbers after LPS challenge were similar irrespective of whether or not mice had received an MI 10 days previous. Viewed together with data on macrophages' roles in regulating hematopoiesis in the bone marrow stem cell niche (Chow et al., 2011; Chow et al., 2013; Hashimoto et al., 2013), these results indicate that after a prior stimulus, bone marrow macrophages may indeed influence the dampened emergency hematopoiesis after recurrent MI.

To analyze the changes in the bone marrow niche in more detail, we isolated key stromal hematopoietic niche cells with FACS sorting in mice three days after MI and compared niche 
factor expression with naive controls and mice with recurrent MI (Figure 8A). The flow cytometric gating for endothelial and leptin receptor ${ }^{+}\left(\right.$LepR-YFP $\left.^{+}\right)$stromal cell isolation is shown in Figure 8B. There were no significant differences for kitl, Vcam1, Angpt1 and Cxcl12 expression in LepR ${ }^{+}$stromal mesenchymal cells or bone marrow endothelial cells in mice with single versus recurrent MI (Figure 8C, D). However, in mice with recurrent MI, bone marrow macrophages expressed significantly more Vcam1 and Angpt1, at levels comparable to naive controls (Figure 8E). Since these factors regulate HSPC retention and quiescence, the data suggest that macrophages contribute to bone marrow tolerance in recurrent Ml. 


\section{Discussion}

Innate immunity and cardiac inflammation have recently been linked to heart failure (Sager et al., 2016; Bajpai et al., 2018; Dick et al., 2019), a connection that may contribute to the dismal survival rate of patients with recurrent MI (Thune et al., 2011). There were no available systems for modeling inflammatory circuits in recurrent MI. We therefore designed a mouse model that allows the induction of two sequential infarctions in different anatomical locations of the same heart. The consequences were characterized by whole-heart fluorescent coronary angiography enabled by optical clearing of the organ and by cardiac MRI. Studying recurrent MI's impact on innate immunity and emergency hematopoiesis, we observed a muted inflammatory response, which is induced by a circulating signal released after the first infarct and possibly mediated by bone marrow macrophages in the hematopoietic niche.

The heart recruits millions of circulating myeloid cells to the ischemic myocardium (Nahrendorf et al., 2007; Leuschner et al., 2012). Because these cells are short lived, with lifespans of about a day (Yona et al., 2013; Patel et al., 2017), the bone marrow augments leukocyte production to meet the organism's need after MI in mice and humans (Dutta et al., 2012; Dutta et al., 2015b). After MI, the marrow is alerted by signals from the sympathetic nervous system (Dutta et al., 2012) and circulating mediators produced by the ischemic heart such as IL-1 $\beta$ and GM-CSF (Sager et al., 2015; Anzai et al., 2017). These mechanisms are not mutually exclusive and can either impact hematopoietic stem cells directly or relay their signals through bone marrow niche cells (Sager et al., 2015). This process controls HSPC quiescence, proliferation and differentiation, and subsequently blood leukocyte levels, in response to myocardial injury. Bone marrow activation is important not only in the context of acute MI. Indeed, chronic inflammatory processes in the cardiovascular system also rely on constant leukocyte supply from the bone marrow, which is stimulated by cardiovascular risk factors such as hyperlipidemia, chronic stress, insufficient sleep, hypertension and diabetes (Swirski et al., 2007; Yvan-Charvet et al., 2010; Nagareddy et al., 2013; Heidt et al., 2014; Al-Sharea et al., 2019; McAlpine et al., 2019).

Cardiovascular mortality correlates with blood leukocyte levels (Ernst et al., 1987; Maekawa et al., 2002; Madjid et al., 2004; Engstrom et al., 2009). As patients with recurrent MI have lower survival rates and aggravated heart failure (Thune et al., 2011), we speculated that recurrent MI would solicit heightened systemic supply of myeloid cells maintained by higher HSPC proliferation rates in the bone marrow. Yet our data indicate that in mice with recurrent MI the opposite is true. Peripheral blood monocytosis and myeloid cell numbers in the heart were lower, and bone marrow activity dimmed after recurrent MI. Clinical data clearly demonstrate that patients with recurrent $\mathrm{MI}$ have a worse prognosis than patients with only one infarct(Thune et al., 2011; Stone et al., 2014); however, information about blood leukocytes, infarct inflammation and bone marrow activity in this cohort are currently unavailable. This should be investigated to compare our findings in mice to human patients.

The potential clinical implications of this work are manifold: if recurrent MI leads to a muted and even insufficient inflammatory response, then perhaps these patients should not receive anti-inflammatory therapeutics after MI. The potential of such therapeutic interventions is currently under active discussion, especially in light of the recent CANTOS trial (Ridker et al., 2017). Prior studies exploring anti-inflammatory therapeutics neutralizing TNFa (Chung et al., 2003; Mann et al., 2004) or nonspecific drugs such as colchicine (Deftereos et al., 2014) returned discouraging results. Careful patient selection, including criteria such as first rather than recurrent MI, may help define a patient subpopulation that benefits from therapeutically dampened hematopoiesis. Determining the optimal inflammatory response required for proper infarct healing may explain how the phenotype we observed in mice links to patients with recurrent MI. If an infarct does not recruit a sufficient number of myeloid cells, wound healing is delayed, because dying cardiomyocytes will not be removed, resulting in more necrotic tissue remnants and lack of granulation tissue and collagen matrix (Nahrendorf et al., 2010). Patients with leukocytosis but also leukopenia have a higher mortality after acute MI (Grzybowski et al., 2004; Coller, 2005). Non-selectively depleting circulating monocytes 
and inhibiting myeloid cell recruitment to the heart impair wound healing and increase left ventricular remodeling after MI in wild type mice (Nahrendorf et al., 2007; van Amerongen et al., 2007). Thus, future studies should examine immune cell levels and hematopoiesis in patients with recurrent MI.

Regarding mechanisms that may attenuate bone marrow activation after recurrent MI, we identified altered expression of niche factors in bone marrow macrophages, which are known to influence HSPC retention, quiescence and differentiation (Chow et al., 2011; Chow et al., 2013; Hashimoto et al., 2013). In our experiments, recurrent MI did not result in a reduction of retention factors that is observed after a first infarct, which induces lower expression of Cxcl12, Vcam1, Scf and Angiopoietin1. Our parabiosis experiments indicate that a circulating, likely heart-derived factor is causally involved. Such signals, which have to be identified in future work, might act directly on macrophages in the bone marrow, where they could induce metabolic and epigenetic rewiring. In "trained immunity", different stimuli induce epigenetic modifications involving histone modifications (Saeed et al., 2014) or metabolic changes that prime myeloid cells and their progenitors for secondary challenges (Cheng et al., 2014; Mitroulis et al., 2018), enabling a higher response after re-stimulation. Other pathways are involved in macrophage tolerance, which is mediated by LPS and requires TLR4 signaling (Foster et al., 2007; Netea et al., 2016). Therefore, it would be interesting to conduct epigenetic screens in bone marrow macrophages to compare the effects of first and recurrent MI. We expected to see an increased immune response in recurrent $\mathrm{MI}$ compared to a first $\mathrm{MI}$, partially because the ischemic myocardium produces IL-1 $\beta$ and GM-CSF (Sager et al., 2015; Anzai et al., 2017), two cytokines that induce HSPC "training" rather than "tolerance" (Christ et al., 2018; Mitroulis et al., 2018). Surprisingly, our data show that $\mathrm{Ml}$ induces "tolerance". Potential candidates inducing such bone marrow tolerance after MI are the TLR4 ligands HMGB1 and S100, which are produced by ischemic myocytes and fibroblasts (Andrassy et al., 2008; Volz et al., 2012; Rohde et al., 2014).

In our studies described here, we examined young and otherwise healthy mice, which likely mount an optimal inflammatory response in response to myocardial injury (Nahrendorf et al., 2010). Increasing or lowering inflammation in these mice has negative effects on infarct healing. Patients with recurrent MI have atherosclerosis and often multiple cardiovascular risk factors, which all may give rise to low grade inflammation (Netea et al., 2017; Ridker et al., 2017). For instance, hyperlipidemia heightens the bone marrow's inflammatory reactions to LPS (Christ et al., 2018). Accordingly, the effects of recurrent MI observed in healthy wild type mice may be different if atherosclerosis and its risk factors are present.

In summary, our data strongly suggest that $\mathrm{Ml}$ alters the innate immune response, which affects systemic inflammation and the organism's capacity to react to subsequent ischemic events and infections. The many preclinical questions arising from our observations can be tackled using the new mouse model of recurrent $\mathrm{MI}$ described in this Resource Article. 


\begin{abstract}
Methods
Animals

C57BL/6 (Jax No. 000664) and B6.SJL-Ptprca Pepcb/BoyJ (Jax No. 002014) were purchased from Jackson Laboratory. B6.129(Cg)-Leprtm2(cre)Rck/J (Jax No. 008320) and B6.129X1-Gt(ROSA)26Sortm1(EYFP)Cos/J (Jax No. 006148 ) were bred in house to generate stromal cell reporter mice. Genotyping for each strain was performed as described on the Jackson Laboratory website. 10-12-week old mice were used for experiments. All mice were maintained and bred in the pathogen-free environment of the Massachusetts General Hospital animal facility. Age-matched female mice were randomly allocated to either control

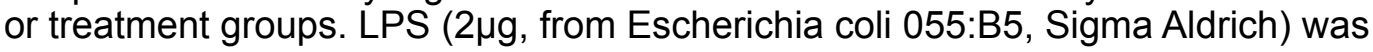
administered intraperitoneally to mice that either had or had not previously received myocardial infarction surgery of the LAD. Clodronate and control liposomes were purchased from Liposoma Research. Depletion studies were performed by intraperitoneally injecting $100 \mu \mathrm{L}$ of liposome formulation per mouse as recommended by the manufacturer.
\end{abstract}

\title{
Procedures to induce myocardial ischemia
}

To permanently ligate the left anterior descending coronary artery (LAD), we followed previously published protocols(Eberli et al., 1998; Lutgens et al., 1999). Mice were anesthetized with $1.5-2 \%$ isoflurane supplemented with oxygen, intubated and ventilated (Inspira, Harvard Apparatus). After thoracotomy, the heart was exposed, and the left coronary artery was identified, and permanently ligated with a monofilament nylon 8-0 suture. The rib cage was then closed using two separate 5-0 sutures, with a $20 \mathrm{G}$ sheath left between the sutures. Finally, air was removed from the thoracic cavity via the sheath and a 3 $\mathrm{ml}$ syringe prior to extubation.

To permanently ligate the circumflex artery (LCX), the heart was exposed and the first artery branching off the LAD was located. If the LCX was not visible, which occurred in about half the cases, the ligation was conducted lateral of the LAD in close proximity to the left atrium. Successful induction of myocardial infarction was verified by identifying a pale ischemic area after ligation.

To induce recurrent MI, mice first received a LCX followed by a LAD ligation 10 days later. The chest was reopened and adhesions were carefully detached. The LAD was identified and permanently ligated, followed by chest cavity closure as described above. In mice subjected to ischemia-reperfusion injury, the coronary artery was ligated for 30 minutes, followed by ligature removal.

\section{Parabiosis}

Parabiosis surgery was performed as previously described(Robbins et al., 2013). In brief, after shaving the corresponding lateral aspects of a CD45.1 and a CD45.2 mouse, matching skin incisions were made in each mouse from behind the ear to the tail, and the subcutaneous fascia were bluntly dissected to create $0.5 \mathrm{~cm}$ of free skin. The left olecranon of one mouse was sutured to the right olecranon of the other mouse with 3.0 non-absorbable suture. Afterwards, the left knee joint of the first mouse was connected to the right knee joint of the second mouse in the same way. Finally, the dorsal and ventral skins were approximated by continuous suture with a 5.0 absorbable Vicryl suture. Mice were joined for two weeks. The CD45.2 mouse was then subjected to MI. After five days, parabionts were surgically split and the CD45.1 mouse received MI surgery five days later.

\section{Optical clearing and fluorescence coronary angiography (FCA)}

For in vivo vasculature labeling, mice were anesthetized with $1.5-2 \%$ isoflurane supplemented with oxygen and sacrificed by perfusion through the left ventricle with $20 \mathrm{~mL}$ of ice-cold PBS followed by $20 \mathrm{~mL}$ of $4 \%$ formaldehyde solution in PBS (Thermo Scientific, Waltham, MA, USA). A $2 \%(\mathrm{w} / \mathrm{v})$ solution $(10 \mathrm{~mL})$ of porcine skin gelatin was prepared in boiling PBS. After the solution reached a temperature below $40^{\circ} \mathrm{C}$, it was combined with a $0.5 \%(\mathrm{w} / \mathrm{v})$ fluorescein-albumin, filtered and injected in the mouse left ventricle(Tsai et al., 
2009). After perfusion the heart was resected and placed in an ice-cold PBS solution for one hour. The heart was then fixed for one hour in a $4 \%$ formaldehyde solution, before being washed for $30 \mathrm{~min}$ in PBS and imaged. Whole heart optical clearing was then performed using the CUBIC (clear, unobstructed brain imaging cocktails and computational analysis) method(Susaki et al., 2014). Hearts were immersed for one day in a chemical mixture obtained by mixing 25 wt\% urea (U16-3, Fisher Scientific Hampton, NH, USA), 25 wt\% N,N,NO,N0-tetrakis(2-hydroxypropyl) ethylenediamine (50-014-48142, Fisher Scientific) and 15 wt\% Triton X-100 (85111, Life Technologies, Carlsbad, CA, USA) at room temperature. Hearts were imaged using a 2x objective with a confocal microscope (FV1000, Olympus America). To visualize the vasculature network along the curvature of the heart, images were displayed as maximum intensity projection of z-stack datasets.

\section{MRI}

MRI was carried out in naive mice and after permanent LCX and LAD ligations at the indicated time points (see Figure 1G) as previously described(Panizzi et al., 2010; Sager et al., 2015). We obtained cine images of the left ventricular short axis by using a 4.7 Tesla horizontal bore Pharmascan (Bruker) and a custom-built mouse cardiac coil (Rapid Biomedical) and a cine gradient echo FLASH-sequence with IntraGate, a self gating software to compensate respiratory and cardiac motion. Acquisition parameters were: echo time: $2.94 \mathrm{~ms}$, repetition time $12 \mathrm{~ms}$, matrix size $200 \times 200 \times 1$, voxel size $0.15 \mathrm{~mm} \times 0.15$ $\mathrm{mm} \times 1 \mathrm{~mm}$, oversampling 350 and 16 cine frames. Delayed enhancement imaging was done 10-20 minutes after i.v. injection of $0.3 \mathrm{mmol} / \mathrm{kg}$ Gd-DTPA at the day 3 timepoint. Images were analyzed using the software Horos (http:// https://horosproject.org). Left ventricular volume, ejection fraction and infarct size were acquired and calculated as previously(Panizzi et al., 2010; Sager et al., 2015).

\section{TTC staining}

Hearts were harvested and sectioned with a heart slicer (zivic instruments). Slices were then incubated in a solution of $2 \%$ TTC (2,3,5-Triphenyltetrazolium chloride, Sigma-Aldrich) for 15 minutes protected from light. ImageJ software was used to quantitate infarct size (imagej.nih.gov).

\section{Tissue processing}

Peripheral blood was collected by retro-orbital bleeding using heparinized capillary tubes (BD Biosciences). For flow cytometry analysis, red blood cells were lysed with $1 \mathrm{x}$ red blood cell lysis buffer (BioLegend). For organ harvest, mice were perfused through the left ventricle with $20 \mathrm{~mL}$ of ice-cold PBS. After harvest, bone marrow for qPCR analysis was isolated by centrifugation. The metaphysis of one end of the tibia was removed, and the bone was spun at $6000 \mathrm{~g}$ with this 'open end' facing down. For qPCR analysis, bone marrow was stored in RLT buffer (Qiagen) at $-80^{\circ} \mathrm{C}$ for further analysis. For flow cytometry analysis of HSPC and mature leukocytes, bone marrow from the femurs was flushed with FACS buffer (1x PBS supplemented with $0.5 \%$ BSA). Infarct heart tissue was minced into small pieces and subjected to enzymatic digestion with $450 \mathrm{U} / \mathrm{mL}$ collagenase I, $125 \mathrm{U} / \mathrm{mL}$ collagenase $\mathrm{XI}, 60$ $\mathrm{U} / \mathrm{mL}$ DNase I and $60 \mathrm{U} / \mathrm{mL}$ hyaluronidase (all Sigma-Aldrich) for 30 minutes at $37^{\circ} \mathrm{C}$ under agitation (750rpm). Digested tissues were then triturated and filtered through a $40 \mu \mathrm{m}$ nylon mesh (Falcon), washed and centrifuged to obtain single-cell suspensions. For stromal cell isolations, the bone marrow fraction was digested to isolate endothelial cells and LepR ${ }^{+}$ cells. The bone marrow fraction was isolated from the long bones and pelvis of mice by flushing the bone marrow plug with a syringe into PBS supplemented with $2 \%$ FBS. After the bone marrow plug sank to the bottom of the tube, the supernatant was removed and replaced with the digestion mix: collagenase IV at $1 \mathrm{mg} / \mathrm{ml}$ (Sigma Aldrich, C5138), Dispase at $2 \mathrm{mg} / \mathrm{ml}$ (Gibco by Life technology, 17105-041) and DNAse I (Thermo scientific, 90083) in HBSS buffer (Gibco by Life Technology, 14025-092). The bone marrow plug was digested 3 $x 15$ minutes at $37^{\circ} \mathrm{C}$. Bone stromal cells in suspension were washed with PBS supplemented with $2 \%$ FBS.

\section{Flow cytometry}


All single cell suspensions were stained at $4^{\circ} \mathrm{C}$ in $300 \mu$ FACS buffer (1x PBS supplemented with $0.5 \% \mathrm{BSA}$ ). For HSPC staining, isolated bone marrow cells were first stained with biotin-conjugated anti-mouse antibodies directed against mouse hematopoietic lineage markers, including CD3 (100304), CD4 (100404), CD8 (100704), CD49b (108904), CD90.2 (105304), CD19 (115503), B220 (103204), NK1.1 (108704), TER119 (116204), CD11b (101204), CD11c (117304), Gr1 (108404; all 1:300, BioLegend). This was followed by a second staining with antibodies for CD16/32-BV711 (101337), CD34-FITC (553733, BD Biosciences), CD135-PE (135306), CD48-AF700 (103426), CD115-BV421 (135513), CD150-PerCP/Cy5.5 (115922), c-kit-PE/Cy7 (105814), Sca-1-BV605 (108133), streptavidinAPC/Cy7 (405208; all 1:150, BioLegend unless otherwise indicated) and, where applicable, BrdU-APC (1:50, BD Biosciences).

For cardiac leukocyte staining, cells were stained with PE-conjugated mouse hematopoietic lineage markers, including B220 (103208), CD103 (121406), CD19 (115508), CD3 (100206), CD49b (108908), CD90.2 (140308), NK1.1 (108708) and Ter119 (116208; all 1:300, BioLegend) as well as CD11b-APC $(1: 300,101212)$, CD45-BV711 $(1: 300,103147)$, F4/80PECy7 (1:150, 123114), Ly-6C-BV605 (1:300, 128035) and Ly-6G-FITC (1:300, 127605, all BioLegend). To stain mature leukocytes in the bone marrow, cells were stained with B220APC/Cy7 (1:300, 103223), CD3-APC/Cy7 (1:300, 100221), Gr1-PE (1:300, 108407), CD115BV605 (1:300, 135517) and F4/80 APC (1:150, Cl A3-1, AbD Serotec). Isotype controls for the APC antibody were used to set the gate for F4/80pos macrophages. To profile macrophages in the bone marrow, we used antibodies for VCAM1-PerCP/Cy5.5 (1:150, 105715), CX3CR1-PerCPCy5.5 (1:300,149009), I-A/I-E-PacificBlue (1:150, 107619) and CD169-PeCy7(1:150, 142411). Respective isotype controls were used. All BioLegend unless otherwise indicated. For blood leukocyte staining, cells were stained with CD19-BV711 (11555), CD45.2-APC-Cy7 (109803) or CD45.1-APC-Cy7 (110715), CD90.2-PE (140308), Ly-6C-BV605 (128035), Ly-6G-FITC (127605), CD11b-APC (101212), CD115-BV421 (135513) and CX3CR1-PerCP (149009, all 1:300, BioLegend ). Blood and bone marrow leukocyte staining samples were fixed with BD Cytofix (BD Biosciences) and analyzed within $24 \mathrm{hrs}$. BrdU staining (552598, BD Biosciences) was done according to the manufacturer's protocol.

\section{Gating strategy for flow cytometry}

All cells were pre-gated on single cells (FSC-A vs FSC-W, and SSC-A vs SSC-W). LSK were identified as $\mathrm{Lin}^{-} \mathrm{C}-\mathrm{kit}^{+} \mathrm{Sca}-1^{+}$. These were further divided into long-term hematopoietic stem cells (LT-HSCs; Lin- c-kit ${ }^{+}$Sca-1+ CD150+ CD48-) and multipotent progenitors (MPPs; Lin- c$\mathrm{kit}^{+} \mathrm{Sca}-1^{+}, \mathrm{CD} 135-\mathrm{CD} 150-\mathrm{CD}^{+} 8^{+}$). Granulocyte macrophage progenitors (GMP) were identified as Lin- c-kit ${ }^{+}$Sca-1- CD16/32+ CD34+. Marrow monocytes were identified as CD3B220- $\mathrm{Gr} 1{ }^{+} \mathrm{CD} 115^{+}$, marrow neutrophils as CD3-B220-, Gr1+CD115- and bone marrow macrophages as CD3- B220- Gr1-CD115- F4/80+ SSClo. Blood monocytes were identified as CD45+ CD19- CD90.2- Ly-6G- CD11b+ CD115+ and neutrophils as CD19- CD90.2- CD115CD11 b+ Ly-6G+. Leptin receptor $\left(\right.$ LepR $\left.^{+}\right)$cells were identified as CD41-CD45- Ter119- Sca1CD31-YFP+ in the bone marrow of LeptinRcre-EYFP mice. Endothelial cells were identified as CD41-CD45- Ter119- CD31+ Sca1+ in the bone marrow of LepR-YFP mice. For compensation, the aforementioned antibodies were conjugated to OneComp eBeads (Affymetrix Inc). Unstained and $\mathrm{YFP}^{+}$control samples were used for compensation and to control the gating strategy. All data were acquired on an LSRII (BD Biosciences) and analyzed with FlowJo software.

\section{Cell sorting}

To purify bone marrow stromal cells, samples were stained with CD31-PE (102507), CD41PerCP (133917), CD45.2-BV605 (103116), Ter119-APC/Cy7 (116223) and Sca1-PeCy7 (108113, all 1:100, Biolegend) and FAC-sorted with a FACSAria II cell sorter (BD Biosciences). For bone marrow macrophages, the same staining panel was used as for bone marrow leukocytes. All cells were pre-gated on single cells (as determined by FSC-A vs FSC-W, and SSC-A vs SSC-W) and viable cells (FxCycle ${ }^{\mathrm{TM}}$ Violet Stain ${ }^{-}$). 


\section{Real-time qPCR}

Total RNA from bone marrow was extracted using the RNeasy Mini kit (Qiagen) according to the manufacturer's protocol. For bone marrow stromal cells, the NucleoSpin RNA XS kit (Takara Bio) was used. RNA $(1 \mu \mathrm{g})$ was reverse-transcribed using the High-Capacity RNA-tocDNA kit (Qiagen) according to the manufacturer's instructions. For stromal cells, all isolated RNA was transcribed. TaqMan gene expression assays were used to quantify target genes. The relative changes were normalized to Gapdh mRNA levels using the $2^{-\Delta \Delta C T}$ method.

\section{Enzyme-linked immunosorbent assay}

For troponin-I ELISA (Life diagnostics, CTNI-1-US) measurements, mice were bled retroorbitally $24 \mathrm{~h}$ after induction of myocardial infarction. Blood plasma was obtained by centrifugation. Plasma was $4 x$ diluted. Samples were analyzed according to the manufacturers' instructions.

\section{Statistical analyses}

Statistical analyses were conducted with GraphPad Prism software Version 8.0. Results are depicted as mean \pm standard error of mean. Data were first tested for normality by

D'Agostino-Pearson omnibus normality test. For a two-group comparison, a Student's t-test was applied if the pretest for normality (D'Agostino-Pearson normality test) was not rejected at the 0.05 significance level; otherwise, a Mann-Whitney $U$ test for nonparametric data was used. To compare more than two groups, an ANOVA test, followed by a Tukey test for multiple comparison, was applied. $P$ values of $<0.05$ indicate statistical significance. No statistical method was used to predetermine sample size and animals were randomly assigned to treatment groups.

\section{Acknowledgements}

This work was funded in part by the National Institutes of Health HL139598, HL125428, HL131495, HL131478, T32HL076136, the European Union's Horizon 2020 Research and Innovation Program under grant agreement No 667837 and the MGH Research Scholar Program. S. Cremer and D. Rohde were funded by Deutsche Forschungsgemeinschaft (CR603/1-1 and RO5071/1-1). The authors declare no competing financial interests. We acknowledge Kaley Joyes for editing the manuscript.

\section{Author contributions}

S. Cremer and M.J. Schloss developed and validated the mouse model of recurrent MI. C. Vinegoni developed fluorescent coronary angiography. S. Cremer, M.J. Schloss, S. Zhang, D. Rohde and C.Vinegoni performed experiments and collected, analyzed and discussed data. G. Wojtkiewicz and S. Schmitt performed imaging experiments and collected data. S. Cremer, F. Swirski and M. Nahrendorf conceived experiments and discussed results and strategy. M. Nahrendorf conceived, designed and directed the study. S. Cremer and M. Nahrendorf wrote the manuscript, which was revised and approved by all authors. 


\section{References}

Ahn, D., L. Cheng, C. Moon, H. Spurgeon, E.G. Lakatta, and M.I. Talan. 2004. Induction of myocardial infarcts of a predictable size and location by branch pattern probability-assisted coronary ligation in C57BL/6 mice. Am J Physiol Heart Circ Physiol. 286:H1201-7. doi: 10.1152/ajpheart.00862.2003.

Al-Sharea, A., M.K.S. Lee, A. Whillas, D.L. Michell, W.A. Shihata, A.J. Nicholls, O.D. Cooney, M.J. Kraakman, C.B. Veiga, A.M. Jefferis, K. Jackson, P.R. Nagareddy, G. Lambert, C.H.Y. Wong, K.L. Andrews, G.A. Head, J. Chin-Dusting, and A.J. Murphy. 2019. Chronic sympathetic driven hypertension promotes atherosclerosis by enhancing hematopoiesis. Haematologica. 104:456-467. doi:10.3324/haematol.2018.192898.

Andrassy, M., H.C. Volz, J.C. Igwe, B. Funke, S.N. Eichberger, Z. Kaya, S. Buss, F. Autschbach, S.T. Pleger, I.K. Lukic, F. Bea, S.E. Hardt, P.M. Humpert, M.E. Bianchi, H. Mairbäurl, P.P. Nawroth, A. Remppis, H.A. Katus, and A. Bierhaus. 2008. High-mobility group box-1 in ischemia-reperfusion injury of the heart. Circulation. 117:3216-3226. doi:10.1161/ CIRCULATIONAHA.108.769331.

Anzai, A., J.L. Choi, S. He, A.M. Fenn, M. Nairz, S. Rattik, C.S. McAlpine, J.E. Mindur, C.T. Chan, Y. Iwamoto, B. Tricot, G.R. Wojtkiewicz, R. Weissleder, P. Libby, M. Nahrendorf, J.R. Stone, B. Becher, and F.K. Swirski. 2017. The infarcted myocardium solicits GM-CSF for the detrimental oversupply of inflammatory leukocytes. J Exp Med. 214:3293-3310. doi:10.1084/ jem.20170689.

Bajpai, G., C. Schneider, N. Wong, A. Bredemeyer, M. Hulsmans, M. Nahrendorf, S. Epelman, D. Kreisel, Y. Liu, A. Itoh, T.S. Shankar, C.H. Selzman, S.G. Drakos, and K.J. Lavine. 2018. The human heart contains distinct macrophage subsets with divergent origins and functions. Nat Med. 24:1234-1245. doi:10.1038/s41591-018-0059-x.

Biswas, S.K., and E. Lopez-Collazo. 2009. Endotoxin tolerance: new mechanisms, molecules and clinical significance. Trends Immunol. 30:475-487. doi:10.1016/j.it. 2009.07.009.

Cheng, S.C., J. Quintin, R.A. Cramer, K.M. Shepardson, S. Saeed, V. Kumar, E.J. Giamarellos-Bourboulis, J.H. Martens, N.A. Rao, A. Aghajanirefah, G.R. Manjeri, Y. Li, D.C. Ifrim, R.J. Arts, B.M. van der Veer, B.M. van der Meer, P.M. Deen, C. Logie, L.A. O'Neill, P. Willems, F.L. van de Veerdonk, J.W. van der Meer, A. Ng, L.A. Joosten, C. Wijmenga, H.G. Stunnenberg, R.J. Xavier, and M.G. Netea. 2014. mTOR- and HIF-1a-mediated aerobic glycolysis as metabolic basis for trained immunity. Science. 345:1250684. doi:10.1126/ science.1250684.

Chow, A., M. Huggins, J. Ahmed, D. Hashimoto, D. Lucas, Y. Kunisaki, S. Pinho, M. Leboeuf, C. Noizat, N. van Rooijen, M. Tanaka, Z.J. Zhao, A. Bergman, M. Merad, and P.S. Frenette. 2013. $\mathrm{CD}_{169^{+}}$macrophages provide a niche promoting erythropoiesis under homeostasis and stress. Nat Med. 19:429-436. doi:10.1038/nm.3057.

Chow, A., D. Lucas, A. Hidalgo, S. Méndez-Ferrer, D. Hashimoto, C. Scheiermann, M. Battista, M. Leboeuf, C. Prophete, N. van Rooijen, M. Tanaka, M. Merad, and P.S. Frenette. 2011. Bone marrow CD169+ macrophages promote the retention of hematopoietic stem and progenitor cells in the mesenchymal stem cell niche. J Exp Med. 208:261-271. doi:10.1084/ jem.20101688.

Christ, A., P. Günther, M.A.R. Lauterbach, P. Duewell, D. Biswas, K. Pelka, C.J. Scholz, M. Oosting, K. Haendler, K. Baßler, K. Klee, J. Schulte-Schrepping, T. Ulas, S.J.C.F.M. Moorlag, V. Kumar, M.H. Park, L.A.B. Joosten, L.A. Groh, N.P. Riksen, T. Espevik, A. Schlitzer, Y. Li, M.L. Fitzgerald, M.G. Netea, J.L. Schultze, and E. Latz. 2018. Western Diet Triggers NLRP3Dependent Innate Immune Reprogramming. Cell. 172:162-175.e14. doi:10.1016/j.cell.

2017.12.013. 
Chung, E.S., M. Packer, K.H. Lo, A.A. Fasanmade, J.T. Willerson, and T.A.C.H.F.I. Anti-TNF. 2003. Randomized, double-blind, placebo-controlled, pilot trial of infliximab, a chimeric monoclonal antibody to tumor necrosis factor-alpha, in patients with moderate-to-severe heart failure: results of the anti-TNF Therapy Against Congestive Heart Failure (ATTACH) trial. Circulation. 107:3133-3140. doi:10.1161/01.CIR.0000077913.60364.D2.

Coller, B.S. 2005. Leukocytosis and ischemic vascular disease morbidity and mortality: is it time to intervene. Arterioscler Thromb Vasc Biol. 25:658-670. doi:10.1161/01.ATV. 0000156877.94472.a5.

Deftereos, S., G. Giannopoulos, V. Panagopoulou, G. Bouras, K. Raisakis, C. Kossyvakis, S. Karageorgiou, C. Papadimitriou, M. Vastaki, A. Kaoukis, C. Angelidis, S. Pagoni, V. Pyrgakis, D. Alexopoulos, A.S. Manolis, C. Stefanadis, and M.W. Cleman. 2014. Anti-inflammatory treatment with colchicine in stable chronic heart failure: a prospective, randomized study. JACC Heart Fail. 2:131-137. doi:10.1016/j.jchf.2013.11.006.

Dick, S.A., J.A. Macklin, S. Nejat, A. Momen, X. Clemente-Casares, M.G. Althagafi, J. Chen, C. Kantores, S. Hosseinzadeh, L. Aronoff, A. Wong, R. Zaman, I. Barbu, R. Besla, K.J. Lavine, B. Razani, F. Ginhoux, M. Husain, M.I. Cybulsky, C.S. Robbins, and S. Epelman. 2019. Self-renewing resident cardiac macrophages limit adverse remodeling following myocardial infarction. Nat Immunol. 20:29-39. doi:10.1038/s41590-018-0272-2.

Dutta, P., G. Courties, Y. Wei, F. Leuschner, R. Gorbatov, C.S. Robbins, Y. Iwamoto, B. Thompson, A.L. Carlson, T. Heidt, M.D. Majmudar, F. Lasitschka, M. Etzrodt, P. Waterman, M.T. Waring, A.T. Chicoine, A.M. van der Laan, H.W. Niessen, J.J. Piek, B.B. Rubin, J. Butany, J.R. Stone, H.A. Katus, S.A. Murphy, D.A. Morrow, M.S. Sabatine, C. Vinegoni, M.A. Moskowitz, M.J. Pittet, P. Libby, C.P. Lin, F.K. Swirski, R. Weissleder, and M. Nahrendorf. 2012. Myocardial infarction accelerates atherosclerosis. Nature. 487:325-329. doi:10.1038/ nature11260.

Dutta, P., F.F. Hoyer, L.S. Grigoryeva, H.B. Sager, F. Leuschner, G. Courties, A. Borodovsky, T. Novobrantseva, V.M. Ruda, K. Fitzgerald, Y. Iwamoto, G. Wojtkiewicz, Y. Sun, N. Da Silva, P. Libby, D.G. Anderson, F.K. Swirski, R. Weissleder, and M. Nahrendorf. 2015a. Macrophages retain hematopoietic stem cells in the spleen via VCAM-1. J Exp Med. 212:497-512. doi:10.1084/jem.20141642.

Dutta, P., H.B. Sager, K.R. Stengel, K. Naxerova, G. Courties, B. Saez, L. Silberstein, T. Heidt, M. Sebas, Y. Sun, G. Wojtkiewicz, P.F. Feruglio, K. King, J.N. Baker, A.M. van der Laan, A. Borodovsky, K. Fitzgerald, M. Hulsmans, F. Hoyer, Y. Iwamoto, C. Vinegoni, D. Brown, M. Di Carli, P. Libby, S.W. Hiebert, D.T. Scadden, F.K. Swirski, R. Weissleder, and M. Nahrendorf. 2015b. Myocardial Infarction Activates CCR2(+) Hematopoietic Stem and Progenitor Cells. Cell Stem Cell. 16:477-487. doi:10.1016/j.stem.2015.04.008.

Eberli, F.R., F. Sam, S. Ngoy, C.S. Apstein, and W.S. Colucci. 1998. Left-ventricular structural and functional remodeling in the mouse after myocardial infarction: assessment with the isovolumetrically-contracting Langendorff heart. J Mol Cell Cardiol. 30:1443-1447. doi:10.1006/jmcc.1998.0702.

Engstrom, G., O. Melander, and B. Hedblad. 2009. Leukocyte count and incidence of hospitalizations due to heart failure. Circ Heart Fail. 2:217-222. doi:10.1161/ CIRCHEARTFAILURE.108.827071.

Ernst, E., D.E. Hammerschmidt, U. Bagge, A. Matrai, and J.A. Dormandy. 1987. Leukocytes and the risk of ischemic diseases. JAMA. 257:2318-2324

Fernández, B., A.C. Durán, M.C. Fernández, T. Fernández-Gallego, J.M. Icardo, and V. Sans-Coma. 2008. The coronary arteries of the C57BL/6 mouse strains: implications for comparison with mutant models. J Anat. 212:12-18. doi:10.1111/j.1469-7580.2007.00838.x. 
Foster, S.L., D.C. Hargreaves, and R. Medzhitov. 2007. Gene-specific control of inflammation by TLR-induced chromatin modifications. Nature. 447:972-978. doi:10.1038/ nature05836.

Frangogiannis, N.G. 2012. Regulation of the inflammatory response in cardiac repair. Circ Res. 110:159-173. doi:10.1161/CIRCRESAHA.111.243162.

Grzybowski, M., R.D. Welch, L. Parsons, C.E. Ndumele, E. Chen, R. Zalenski, and H.V. Barron. 2004. The association between white blood cell count and acute myocardial infarction in-hospital mortality: findings from the National Registry of Myocardial Infarction. Acad Emerg Med. 11:1049-1060. doi:10.1197/j.aem.2004.06.005.

Hashimoto, D., A. Chow, C. Noizat, P. Teo, M.B. Beasley, M. Leboeuf, C.D. Becker, P. See, J. Price, D. Lucas, M. Greter, A. Mortha, S.W. Boyer, E.C. Forsberg, M. Tanaka, N. van Rooijen, A. García-Sastre, E.R. Stanley, F. Ginhoux, P.S. Frenette, and M. Merad. 2013. Tissue-resident macrophages self-maintain locally throughout adult life with minimal contribution from circulating monocytes. Immunity. 38:792-804. doi:10.1016/j.immuni. 2013.04.004.

Heidt, T., H.B. Sager, G. Courties, P. Dutta, Y. Iwamoto, A. Zaltsman, C. von Zur Muhlen, C. Bode, G.L. Fricchione, J. Denninger, C.P. Lin, C. Vinegoni, P. Libby, F.K. Swirski, R. Weissleder, and M. Nahrendorf. 2014. Chronic variable stress activates hematopoietic stem cells. Nat Med. 20:754-758. doi:10.1038/nm.3589.

Kim, R.J., E. Wu, A. Rafael, E.L. Chen, M.A. Parker, O. Simonetti, F.J. Klocke, R.O. Bonow, and R.M. Judd. 2000. The use of contrast-enhanced magnetic resonance imaging to identify reversible myocardial dysfunction. N Engl J Med. 343:1445-1453. doi:10.1056/ NEJM200011163432003.

Leuschner, F., P.J. Rauch, T. Ueno, R. Gorbatov, B. Marinelli, W.W. Lee, P. Dutta, Y. Wei, C. Robbins, Y. Iwamoto, B. Sena, A. Chudnovskiy, P. Panizzi, E. Keliher, J.M. Higgins, P. Libby, M.A. Moskowitz, M.J. Pittet, F.K. Swirski, R. Weissleder, and M. Nahrendorf. 2012. Rapid monocyte kinetics in acute myocardial infarction are sustained by extramedullary monocytopoiesis. J Exp Med. 209:123-137. doi:10.1084/jem.20111009.

Lutgens, E., M.J. Daemen, E.D. de Muinck, J. Debets, P. Leenders, and J.F. Smits. 1999. Chronic myocardial infarction in the mouse: cardiac structural and functional changes. Cardiovasc Res. 41:586-593. doi:10.1016/s0008-6363(98)00216-8.

Madjid, M., I. Awan, J.T. Willerson, and S.W. Casscells. 2004. Leukocyte count and coronary heart disease: implications for risk assessment. J Am Coll Cardiol. 44:1945-1956. doi: 10.1016/j.jacc.2004.07.056.

Maekawa, Y., T. Anzai, T. Yoshikawa, Y. Asakura, T. Takahashi, S. Ishikawa, H. Mitamura, and S. Ogawa. 2002. Prognostic significance of peripheral monocytosis after reperfused acute myocardial infarction:a possible role for left ventricular remodeling. J Am Coll Cardiol. 39:241-246

Mann, D.L., J.J. McMurray, M. Packer, K. Swedberg, J.S. Borer, W.S. Colucci, J. Djian, H. Drexler, A. Feldman, L. Kober, H. Krum, P. Liu, M. Nieminen, L. Tavazzi, D.J. van Veldhuisen, A. Waldenstrom, M. Warren, A. Westheim, F. Zannad, and T. Fleming. 2004. Targeted anticytokine therapy in patients with chronic heart failure: results of the Randomized Etanercept Worldwide Evaluation (RENEWAL). Circulation. 109:1594-1602. doi:10.1161/01.CIR.0000124490.27666.B2.

McAlpine, C.S., M.G. Kiss, S. Rattik, S. He, A. Vassalli, C. Valet, A. Anzai, C.T. Chan, J.E. Mindur, F. Kahles, W.C. Poller, V. Frodermann, A.M. Fenn, A.F. Gregory, L. Halle, Y. Iwamoto, F.F. Hoyer, C.J. Binder, P. Libby, M. Tafti, T.E. Scammell, M. Nahrendorf, and F.K. Swirski. 2019. Sleep modulates haematopoiesis and protects against atherosclerosis. Nature. 566:383-387. doi:10.1038/s41586-019-0948-2. 
Mendelson, A., and P.S. Frenette. 2014. Hematopoietic stem cell niche maintenance during homeostasis and regeneration. Nat Med. 20:833-846. doi:10.1038/nm.3647.

Mitroulis, I., K. Ruppova, B. Wang, L.S. Chen, M. Grzybek, T. Grinenko, A. Eugster, M. Troullinaki, A. Palladini, I. Kourtzelis, A. Chatzigeorgiou, A. Schlitzer, M. Beyer, L.A.B. Joosten, B. Isermann, M. Lesche, A. Petzold, K. Simons, I. Henry, A. Dahl, J.L. Schultze, B. Wielockx, N. Zamboni, P. Mirtschink, Ü. Coskun, G. Hajishengallis, M.G. Netea, and T. Chavakis. 2018. Modulation of Myelopoiesis Progenitors Is an Integral Component of Trained Immunity. Cell. 172:147-161.e12. doi:10.1016/j.cell.2017.11.034.

Morrison, S.J., and D.T. Scadden. 2014. The bone marrow niche for haematopoietic stem cells. Nature. 505:327-334. doi:10.1038/nature12984.

Nagareddy, P.R., A.J. Murphy, R.A. Stirzaker, Y. Hu, S. Yu, R.G. Miller, B. Ramkhelawon, E. Distel, M. Westerterp, L.S. Huang, A.M. Schmidt, T.J. Orchard, E.A. Fisher, A.R. Tall, and I.J. Goldberg. 2013. Hyperglycemia promotes myelopoiesis and impairs the resolution of atherosclerosis. Cell Metab. 17:695-708. doi:10.1016/j.cmet.2013.04.001.

Nahrendorf, M. 2018. Myeloid cell contributions to cardiovascular health and disease. Nat Med. 24:711-720. doi:10.1038/s41591-018-0064-0.

Nahrendorf, M., M.J. Pittet, and F.K. Swirski. 2010. Monocytes: protagonists of infarct inflammation and repair after myocardial infarction. Circulation. 121:2437-2445. doi:10.1161/ CIRCULATIONAHA.109.916346.

Nahrendorf, M., F.K. Swirski, E. Aikawa, L. Stangenberg, T. Wurdinger, J.L. Figueiredo, P. Libby, R. Weissleder, and M.J. Pittet. 2007. The healing myocardium sequentially mobilizes two monocyte subsets with divergent and complementary functions. J Exp Med. 204:3037-3047. doi:10.1084/jem.20070885.

Netea, M.G., F. Balkwill, M. Chonchol, F. Cominelli, M.Y. Donath, E.J. GiamarellosBourboulis, D. Golenbock, M.S. Gresnigt, M.T. Heneka, H.M. Hoffman, R. Hotchkiss, L.A.B. Joosten, D.L. Kastner, M. Korte, E. Latz, P. Libby, T. Mandrup-Poulsen, A. Mantovani, K.H.G. Mills, K.L. Nowak, L.A. O'Neill, P. Pickkers, T. van der Poll, P.M. Ridker, J. Schalkwijk, D.A. Schwartz, B. Siegmund, C.J. Steer, H. Tilg, J.W.M. van der Meer, F.L. van de Veerdonk, and C.A. Dinarello. 2017. A guiding map for inflammation. Nat Immunol. 18:826-831. doi: 10.1038/ni.3790.

Netea, M.G., L.A. Joosten, E. Latz, K.H. Mills, G. Natoli, H.G. Stunnenberg, L.A. O'Neill, and R.J. Xavier. 2016. Trained immunity: A program of innate immune memory in health and disease. Science. 352:aaf1098. doi:10.1126/science.aaf1098.

Panizzi, P., F.K. Swirski, J.L. Figueiredo, P. Waterman, D.E. Sosnovik, E. Aikawa, P. Libby, M. Pittet, R. Weissleder, and M. Nahrendorf. 2010. Impaired infarct healing in atherosclerotic mice with Ly-6C(hi) monocytosis. J Am Coll Cardiol. 55:1629-1638. doi:10.1016/j.jacc. 2009.08.089.

Patel, A.A., Y. Zhang, J.N. Fullerton, L. Boelen, A. Rongvaux, A.A. Maini, V. Bigley, R.A. Flavell, D.W. Gilroy, B. Asquith, D. Macallan, and S. Yona. 2017. The fate and lifespan of human monocyte subsets in steady state and systemic inflammation. $J$ Exp Med. 214:1913-1923. doi:10.1084/jem.20170355.

Richardson, D.S., and J.W. Lichtman. 2015. Clarifying Tissue Clearing. Cell. 162:246-257. doi:10.1016/j.cell.2015.06.067.

Ridker, P.M., B.M. Everett, T. Thuren, J.G. MacFadyen, W.H. Chang, C. Ballantyne, F. Fonseca, J. Nicolau, W. Koenig, S.D. Anker, J.J.P. Kastelein, J.H. Cornel, P. Pais, D. Pella, J. Genest, R. Cifkova, A. Lorenzatti, T. Forster, Z. Kobalava, L. Vida-Simiti, M. Flather, H. Shimokawa, H. Ogawa, M. Dellborg, P.R.F. Rossi, R.P.T. Troquay, P. Libby, R.J. Glynn, and T.G. CANTOS. 2017. Antiinflammatory Therapy with Canakinumab for Atherosclerotic Disease. N Engl J Med. 377:1119-1131. doi:10.1056/NEJMoa1707914. 
Robbins, C.S., I. Hilgendorf, G.F. Weber, I. Theurl, Y. Iwamoto, J.L. Figueiredo, R. Gorbatov, G.K. Sukhova, L.M. Gerhardt, D. Smyth, C.C. Zavitz, E.A. Shikatani, M. Parsons, N. van Rooijen, H.Y. Lin, M. Husain, P. Libby, M. Nahrendorf, R. Weissleder, and F.K. Swirski. 2013. Local proliferation dominates lesional macrophage accumulation in atherosclerosis. Nat Med. 19:1166-1172. doi:10.1038/nm.3258.

Rohde, D., C. Schön, M. Boerries, I. Didrihsone, J. Ritterhoff, K.F. Kubatzky, M. Völkers, N. Herzog, M. Mähler, J.N. Tsoporis, T.G. Parker, B. Linke, E. Giannitsis, E. Gao, K. Peppel, H.A. Katus, and P. Most. 2014. S100A1 is released from ischemic cardiomyocytes and signals myocardial damage via Toll-like receptor 4. EMBO Mol Med. 6:778-794. doi: 10.15252/emmm.201303498.

Saeed, S., J. Quintin, H.H. Kerstens, N.A. Rao, A. Aghajanirefah, F. Matarese, S.C. Cheng, J. Ratter, K. Berentsen, M.A. van der Ent, N. Sharifi, E.M. Janssen-Megens, M. Ter Huurne, A. Mandoli, T. van Schaik, A. Ng, F. Burden, K. Downes, M. Frontini, V. Kumar, E.J. Giamarellos-Bourboulis, W.H. Ouwehand, J.W. van der Meer, L.A. Joosten, C. Wijmenga, J.H. Martens, R.J. Xavier, C. Logie, M.G. Netea, and H.G. Stunnenberg. 2014. Epigenetic programming of monocyte-to-macrophage differentiation and trained innate immunity. Science. 345:1251086. doi:10.1126/science.1251086.

Sager, H.B., T. Heidt, M. Hulsmans, P. Dutta, G. Courties, M. Sebas, G.R. Wojtkiewicz, B. Tricot, Y. Iwamoto, Y. Sun, R. Weissleder, P. Libby, F.K. Swirski, and M. Nahrendorf. 2015. Targeting Interleukin-1 $\beta$ Reduces Leukocyte Production After Acute Myocardial Infarction. Circulation. 132:1880-1890. doi:10.1161/CIRCULATIONAHA.115.016160.

Sager, H.B., M. Hulsmans, K.J. Lavine, M.B. Moreira, T. Heidt, G. Courties, Y. Sun, Y. Iwamoto, B. Tricot, O.F. Khan, J.E. Dahlman, A. Borodovsky, K. Fitzgerald, D.G. Anderson, R. Weissleder, P. Libby, F.K. Swirski, and M. Nahrendorf. 2016. Proliferation and Recruitment Contribute to Myocardial Macrophage Expansion in Chronic Heart Failure. Circ Res. 119:853-864. doi:10.1161/CIRCRESAHA.116.309001.

Stone, S.G., G.W. Serrao, R. Mehran, M.I. Tomey, B. Witzenbichler, G. Guagliumi, J.Z. Peruga, B.R. Brodie, D. Dudek, M. Möckel, S.J. Brener, G. Dangas, and G.W. Stone. 2014. Incidence, predictors, and implications of reinfarction after primary percutaneous coronary intervention in ST-segment-elevation myocardial infarction: the Harmonizing Outcomes with Revascularization and Stents in Acute Myocardial Infarction Trial. Circ Cardiovasc Interv. 7:543-551. doi:10.1161/CIRCINTERVENTIONS.114.001360.

Susaki, E.A., K. Tainaka, D. Perrin, F. Kishino, T. Tawara, T.M. Watanabe, C. Yokoyama, H. Onoe, M. Eguchi, S. Yamaguchi, T. Abe, H. Kiyonari, Y. Shimizu, A. Miyawaki, H. Yokota, and H.R. Ueda. 2014. Whole-brain imaging with single-cell resolution using chemical cocktails and computational analysis. Cell. 157:726-739. doi:10.1016/j.cell.2014.03.042.

Swirski, F.K., P. Libby, E. Aikawa, P. Alcaide, F.W. Luscinskas, R. Weissleder, and M.J. Pittet. 2007. Ly-6Chi monocytes dominate hypercholesterolemia-associated monocytosis and give rise to macrophages in atheromata. J Clin Invest. 117:195-205. doi:10.1172/JCI29950.

Swirski, F.K., M. Nahrendorf, M. Etzrodt, M. Wildgruber, V. Cortez-Retamozo, P. Panizzi, J.L. Figueiredo, R.H. Kohler, A. Chudnovskiy, P. Waterman, E. Aikawa, T.R. Mempel, P. Libby, R. Weissleder, and M.J. Pittet. 2009. Identification of splenic reservoir monocytes and their deployment to inflammatory sites. Science. 325:612-616. doi:10.1126/science.1175202.

Thune, J.J., J.E. Signorovitch, L. Kober, J.J. McMurray, K. Swedberg, J. Rouleau, A. Maggioni, E. Velazquez, R. Califf, M.A. Pfeffer, and S.D. Solomon. 2011. Predictors and prognostic impact of recurrent myocardial infarction in patients with left ventricular dysfunction, heart failure, or both following a first myocardial infarction. Eur J Heart Fail. 13:148-153. doi:10.1093/eurjhf/hfq194.

Tsai, P.S., J.P. Kaufhold, P. Blinder, B. Friedman, P.J. Drew, H.J. Karten, P.D. Lyden, and D. Kleinfeld. 2009. Correlations of neuronal and microvascular densities in murine cortex 
revealed by direct counting and colocalization of nuclei and vessels. $J$ Neurosci. 29:14553-14570. doi:10.1523/JNEUROSCI.3287-09.2009.

van Amerongen, M.J., M.C. Harmsen, N. van Rooijen, A.H. Petersen, and M.J. van Luyn. 2007. Macrophage depletion impairs wound healing and increases left ventricular remodeling after myocardial injury in mice. Am J Pathol. 170:818-829. doi:10.2353/ajpath. 2007.060547.

Volz, H.C., D. Laohachewin, C. Seidel, F. Lasitschka, K. Keilbach, A.R. Wienbrandt, J. Andrassy, A. Bierhaus, Z. Kaya, H.A. Katus, and M. Andrassy. 2012. S100A8/A9 aggravates post-ischemic heart failure through activation of RAGE-dependent NF-KB signaling. Basic Res Cardiol. 107:250. doi:10.1007/s00395-012-0250-z.

Yang, Z., S.S. Berr, W.D. Gilson, M.C. Toufektsian, and B.A. French. 2004. Simultaneous evaluation of infarct size and cardiac function in intact mice by contrast-enhanced cardiac magnetic resonance imaging reveals contractile dysfunction in noninfarcted regions early after myocardial infarction. Circulation. 109:1161-1167. doi:10.1161/01.CIR. 0000118495.88442.32.

Yona, S., K.W. Kim, Y. Wolf, A. Mildner, D. Varol, M. Breker, D. Strauss-Ayali, S. Viukov, M. Guilliams, A. Misharin, D.A. Hume, H. Perlman, B. Malissen, E. Zelzer, and S. Jung. 2013. Fate mapping reveals origins and dynamics of monocytes and tissue macrophages under homeostasis. Immunity. 38:79-91. doi:10.1016/j.immuni.2012.12.001.

Yvan-Charvet, L., T. Pagler, E.L. Gautier, S. Avagyan, R.L. Siry, S. Han, C.L. Welch, N. Wang, G.J. Randolph, H.W. Snoeck, and A.R. Tall. 2010. ATP-binding cassette transporters and HDL suppress hematopoietic stem cell proliferation. Science. 328:1689-1693. doi: 10.1126/science.1189731. 


\section{Figure legends}
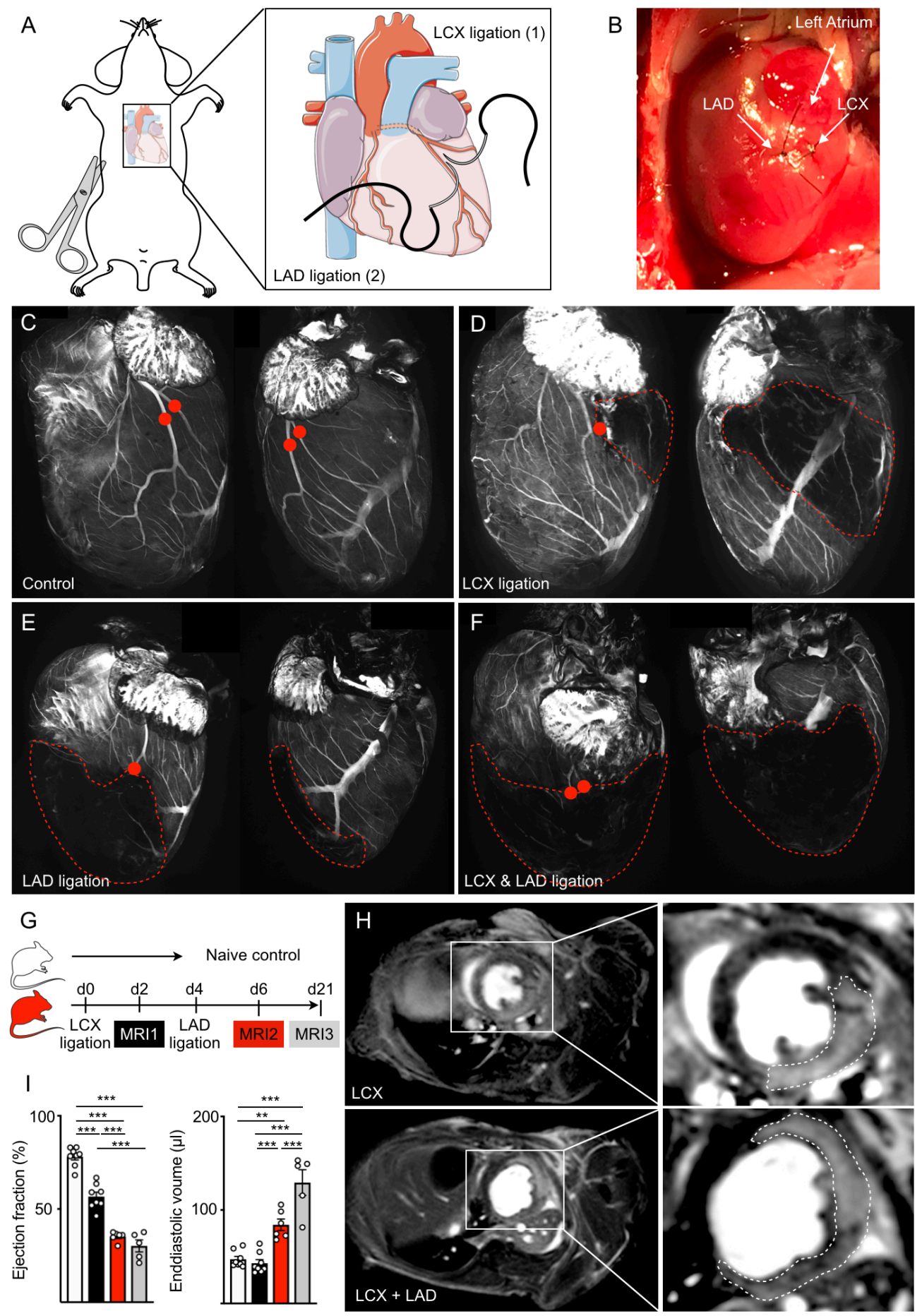

Figure 1. Recurrent MI in mice. (A) Surgical approach. The LAD and its first branch (LCX) are ligated separately. (B) Image of LAD and LCX ligations in the same heart. (C) Confocal projections of the coronary vasculature after optical clearing and in vivo staining with fluorescein albumin in naive mice, (D) mice with LCX and (E) LAD infarctions and (F) after ligation of both vessels. Red dots indicate the ligation sites; the infarcted area is outlined. (G) Timeline of MRI. (H) MRI delayed enhancement short axis views of a mouse with LCX MI (upper panel) and the same mouse after subsequent LAD MI (lower panel). Insets show magnification views of the left ventricle. The MI is indicated by a dotted line. (I) Ejection fraction and end-diastolic volume, assessed by MRI, of naive mice and mice after one or two Mls and three weeks thereafter. ${ }^{* *} \mathrm{p}<0.01,{ }^{* * *} \mathrm{p}<0.001, \mathrm{n}=5-8$, one-way analysis of variance (ANOVA) followed by Tukey's multiple comparisons test. Data are mean \pm s.e.m.. 


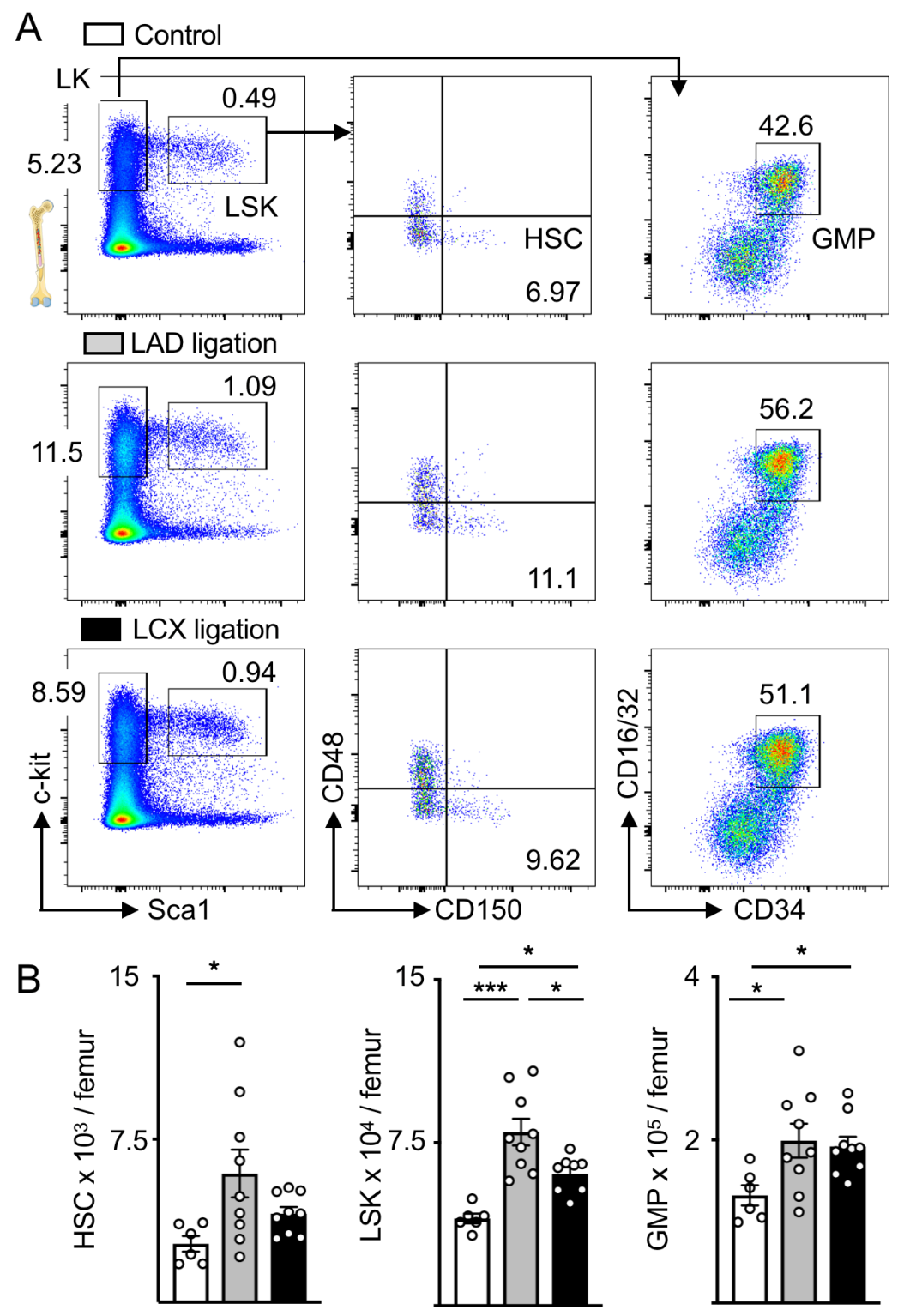

Figure 2. LCX and LAD ligation activate the bone marrow. (A) Dot plots of hematopoietic stem and progenitor cells (LT-HSC, LSK and GMP) in naive mice, mice three days after LAD or LCX ligation. (B) Quantification of cell numbers of LT-HSC, LSK and GMP. ${ }^{*} p<0.05,{ }^{* * *} p<$ $0.001, n=6-9$, one-way analysis of variance (ANOVA) followed by Tukey's multiple comparisons test. Data are mean \pm s.e.m.. 
A
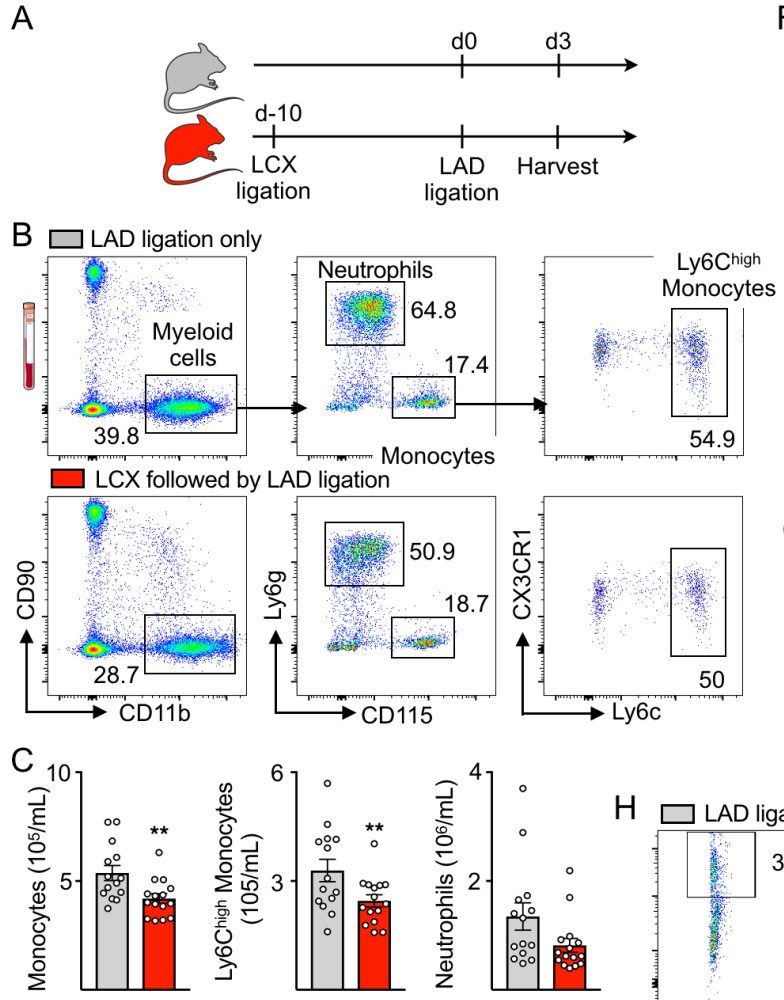

$\mathrm{D}$

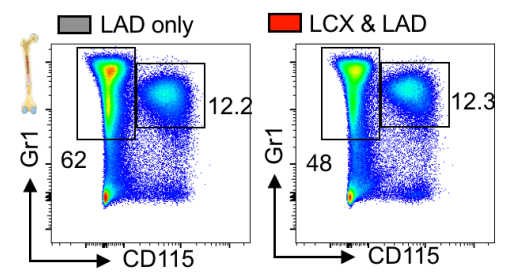

E

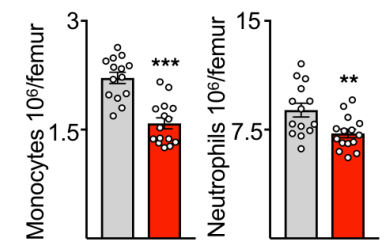

$\mathrm{H} \square$ LAD ligation only
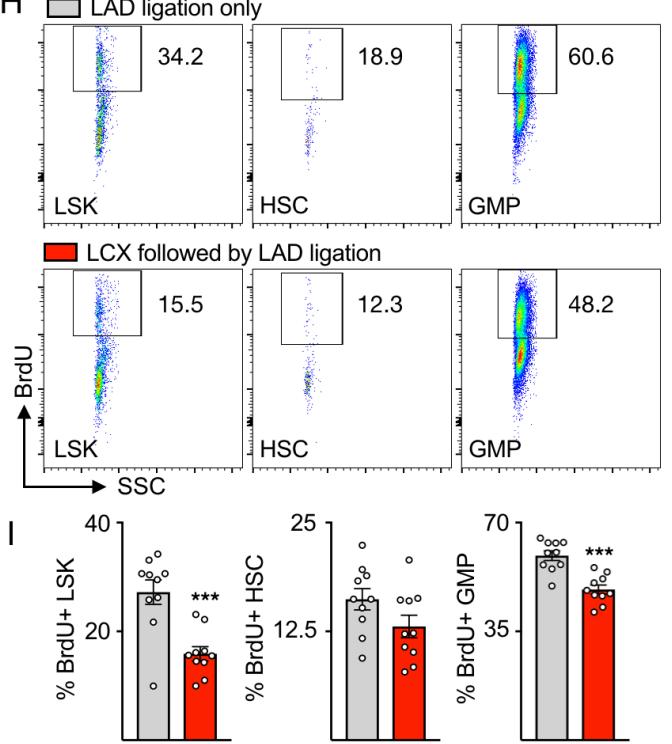

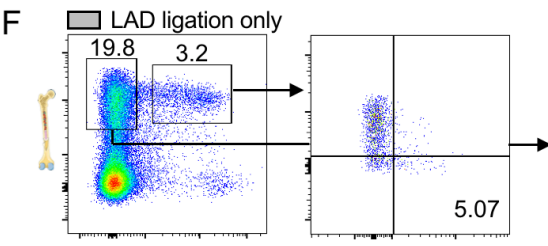

$\square$ LCX followed by LAD ligation
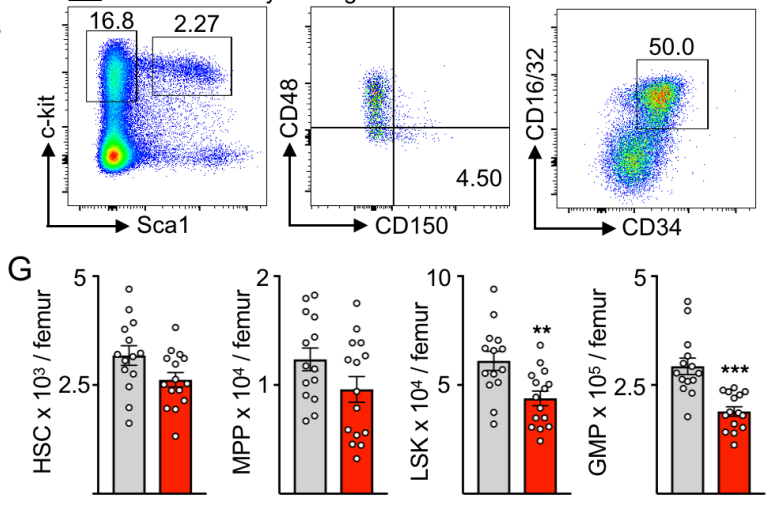

Figure 3. Attenuated systemic inflammation in recurrent MI. (A) Experimental outline. Mice were subjected to LCX MI 10 days before LAD MI. Mice were analyzed on day three after LAD MI. (B) Dot plots and (C) quantification of blood myeloid cells from mice with only one MI compared to recurrent MI. (D) Dot plots and (E) quantification of neutrophils and monocytes in bone marrow. (F) Flow plots and (G) quantification of HSPC in bone marrow. (H) Dot plots and (I) quantification of BrdU incorporation for HSPC proliferation. (J) Gene expression by qPCR in total bone marrow. mRNA levels were normalized to gapdh $\mathrm{Ct}$ values. ${ }^{*} p<0.05,{ }^{* *} p<0.01,{ }^{* * *} p<0.001, n=9-15$, Student's t-test. Data are mean \pm s.e.m.. 
A
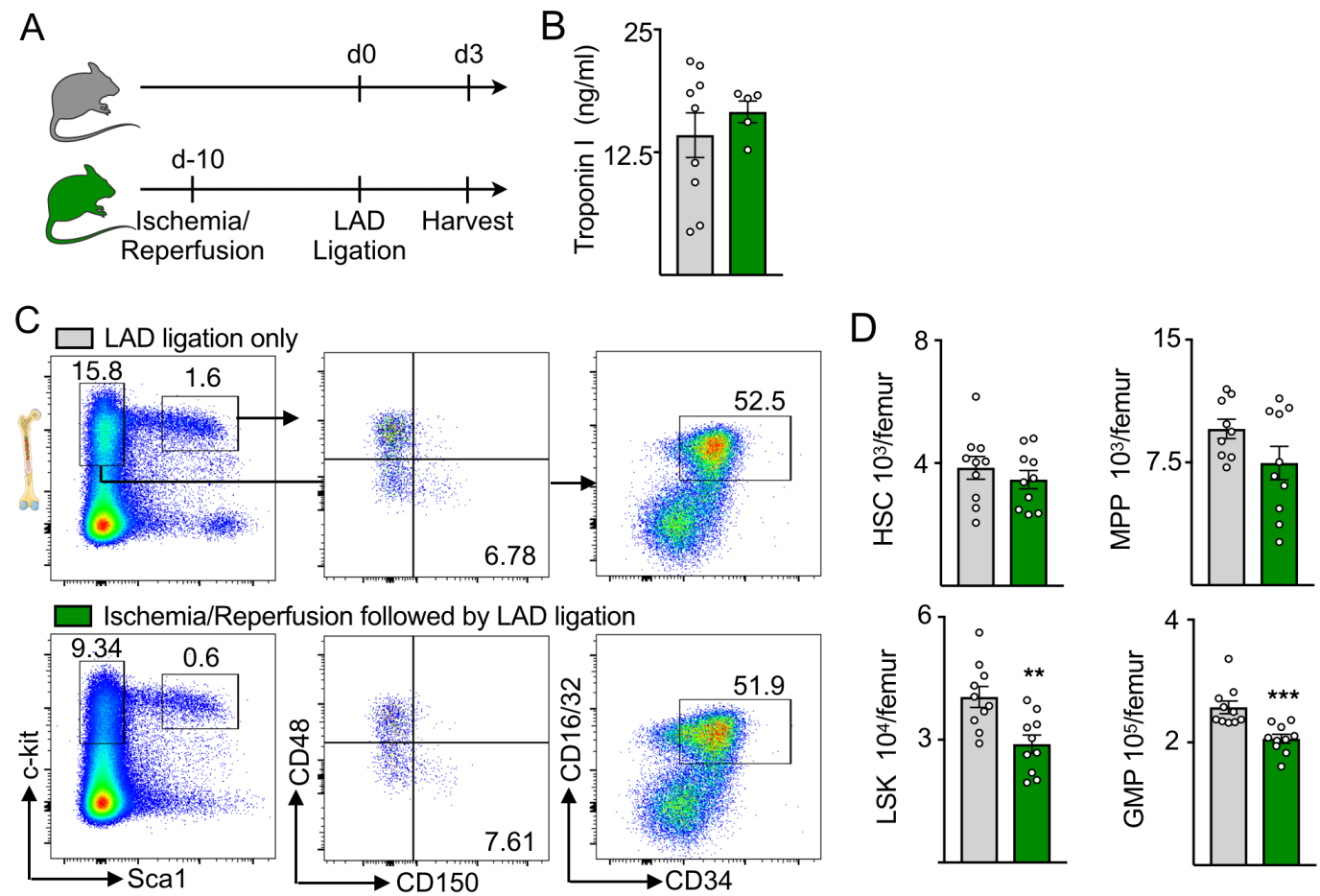

$\mathrm{E} \square$ LAD ligation only
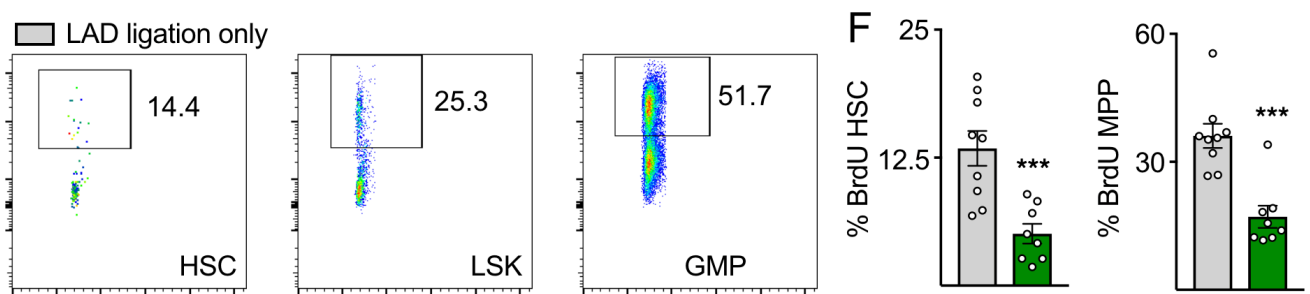

Ischemia/Reperfusion followed by LAD Ligation
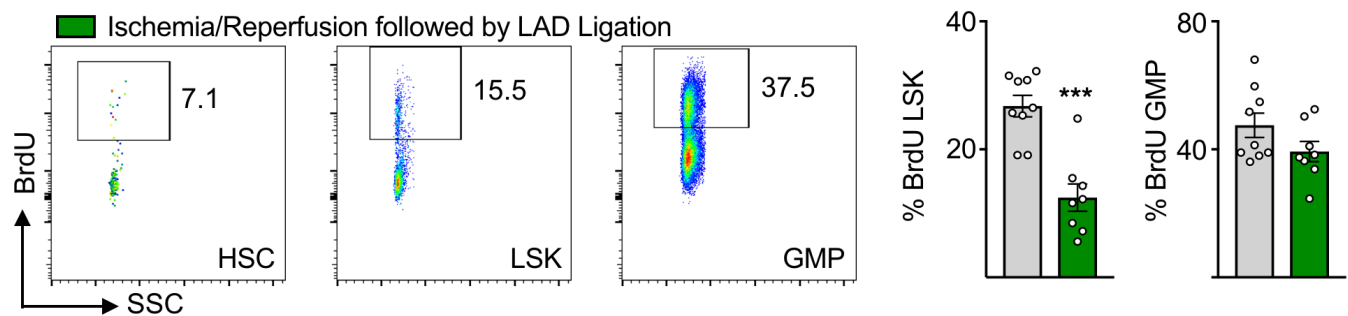

Figure 4. Previous ischemia reperfusion injury (IRI) restricts bone marrow activity after MI. (A) Experimental design. Mice were subjected to $30 \mathrm{~min}$ ischemia by temporary LAD ligation followed by reperfusion 10 days before inducing LAD MI. Mice were analyzed on day three after LAD MI. (B) Troponin-I values 24 hours after LAD MI ( $n=4-9)$. (C) Flow plots and (D) quantification of HSPC numbers in bone marrow. (E) Dot plots and (F) quantification of BrdU incorporation assay for bone marrow HSPC. ${ }^{* *} p<0.01,{ }^{* * *} p<0.001$, $n=8-10$, Student's t-test. Data are mean \pm s.e.m.. 


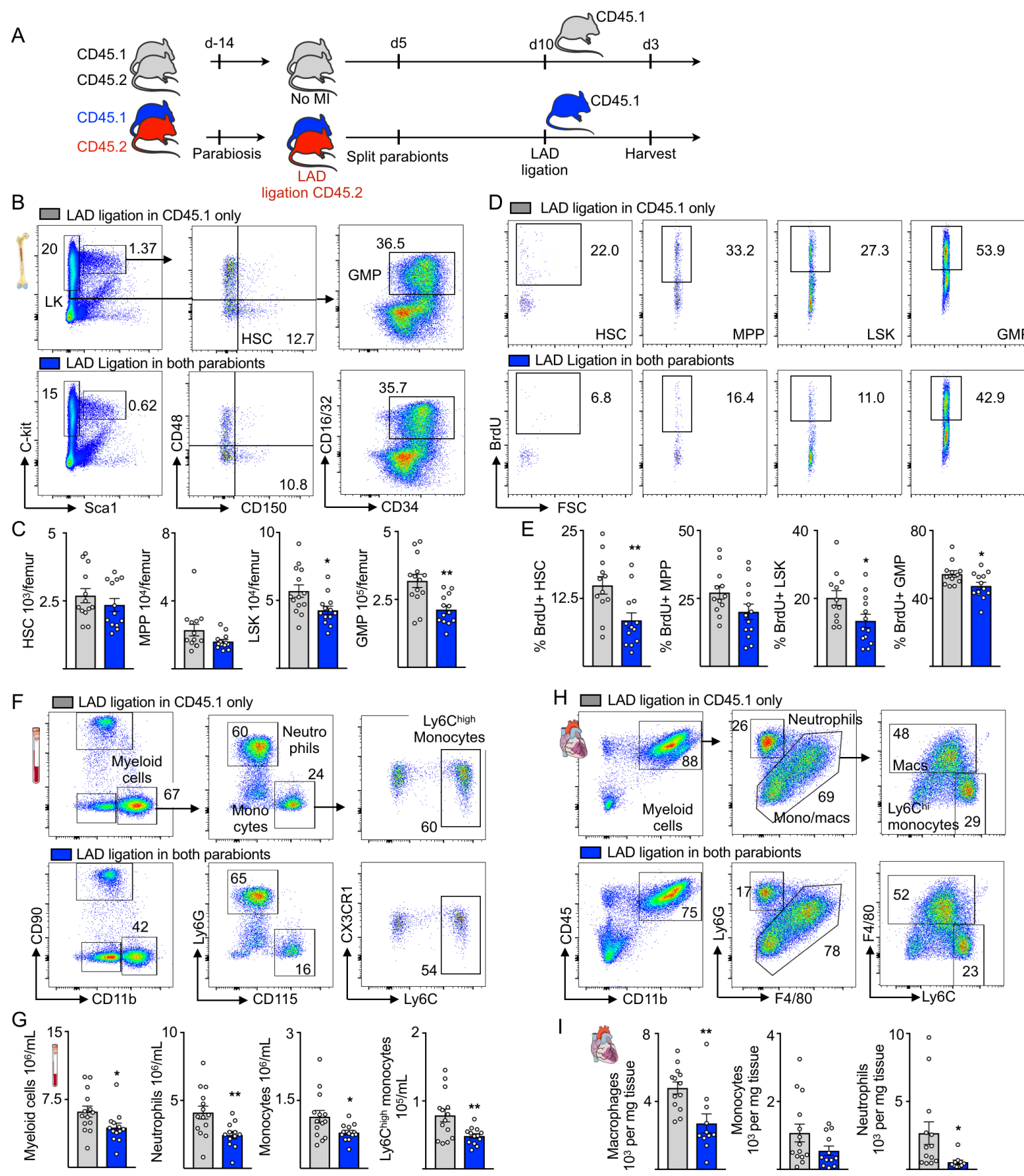

Figure 5. A circulating factor attenuates progenitor cell expansion. (A) Experimental design. Mice were joined in parabiosis. Two weeks later one mouse received MI surgery. Five days later parabionts were separated, and LAD ligation was conducted in the other mouse five days after. (B) Dot plots and (C) quantification of bone marrow HSPC. (D) Dot plots and (E) quantification of BrdU incorporation assay for bone marrow HSPC. (F) Dot plots and (G) quantification of blood myeloid cells. (H) Flow cytometry gating and (I) quantification of leukocyte subsets from infarcted myocardium. ${ }^{*} p<0.05,{ }^{* *} p<0.01, n=12-14$, Student's t-test. Data are mean \pm s.e.m.. 
A

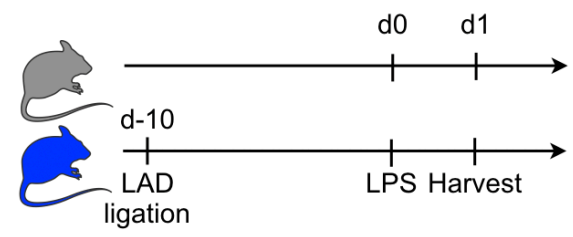

C

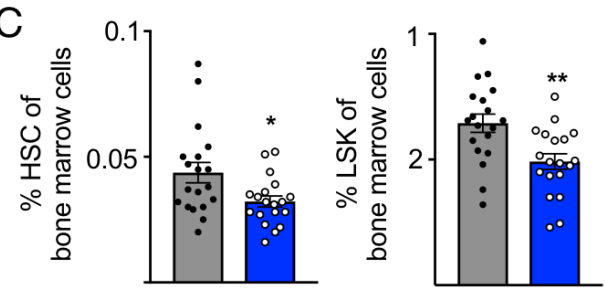

$\mathrm{B}$

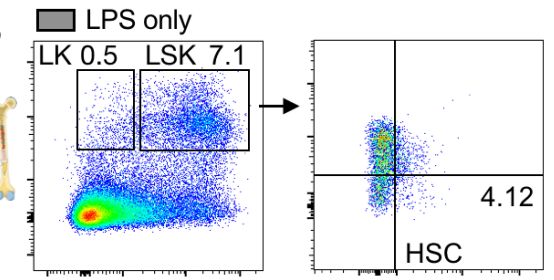

$\square$ LAD Ligation + LPS

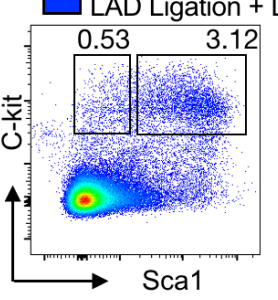

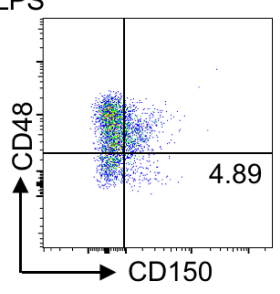

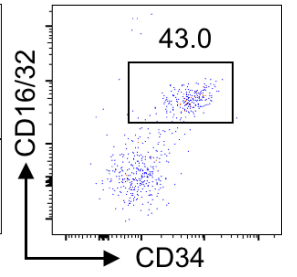

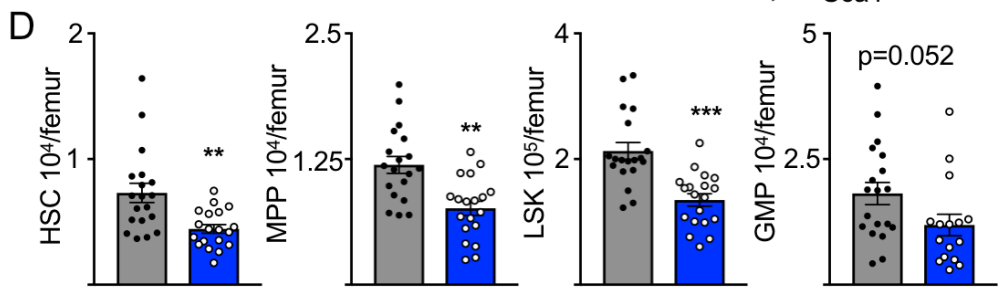
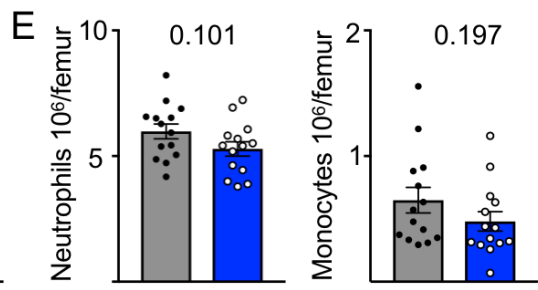

$\mathrm{F}$
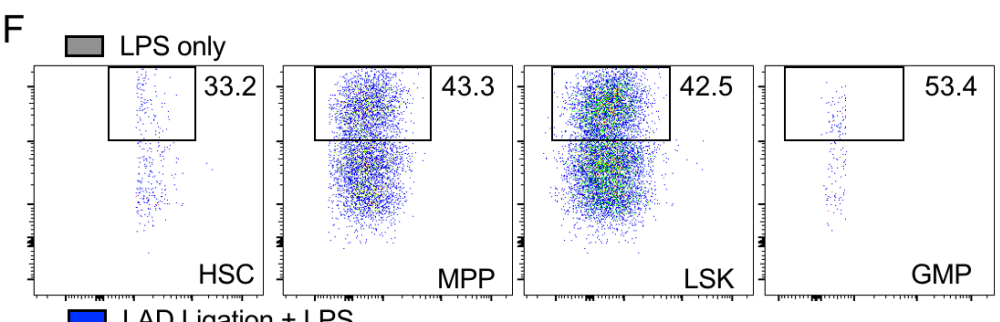

\section{G}
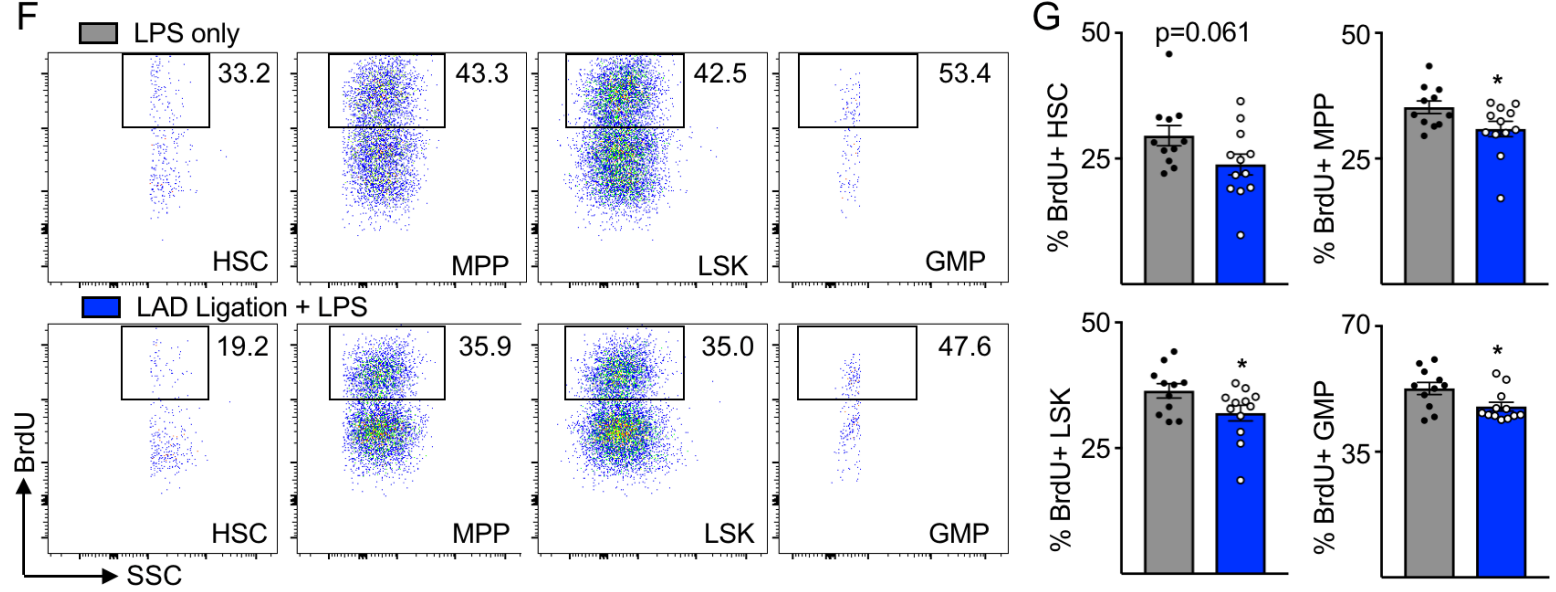

Figure 6. Prior MI attenuates HSPC expansion after LPS. (A) Experimental design. Mice received $\mathrm{MI}$ surgery 10 days before sepsis induction by i.p. LPS injection. (B) Flow plots, (C) frequencies and (D) quantification of bone marrow HSPC. (E) Leukocytes in bone marrow. (F) Representative dot plots and (G) quantification of BrdU incorporation for bone marrow HSPC. ${ }^{*} p<0.05,{ }^{* *} p<0.01,{ }^{* * *} p<0.001, n=12-14$, Student's t-test. Data are mean \pm s.e.m. 
A
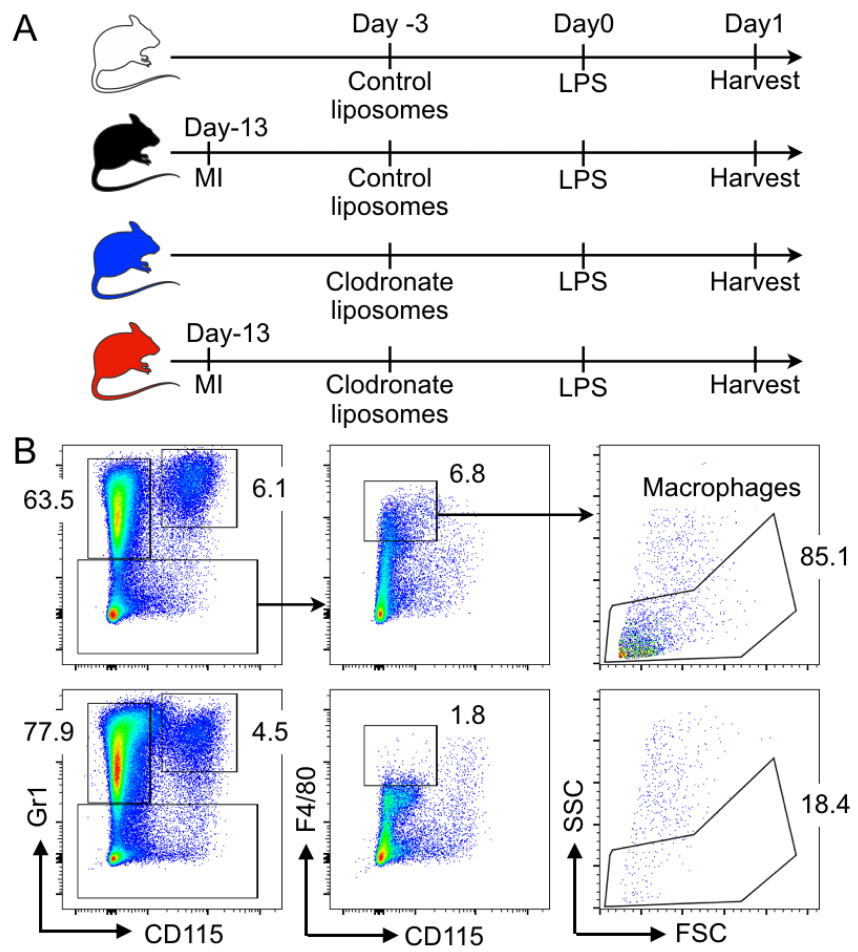

C
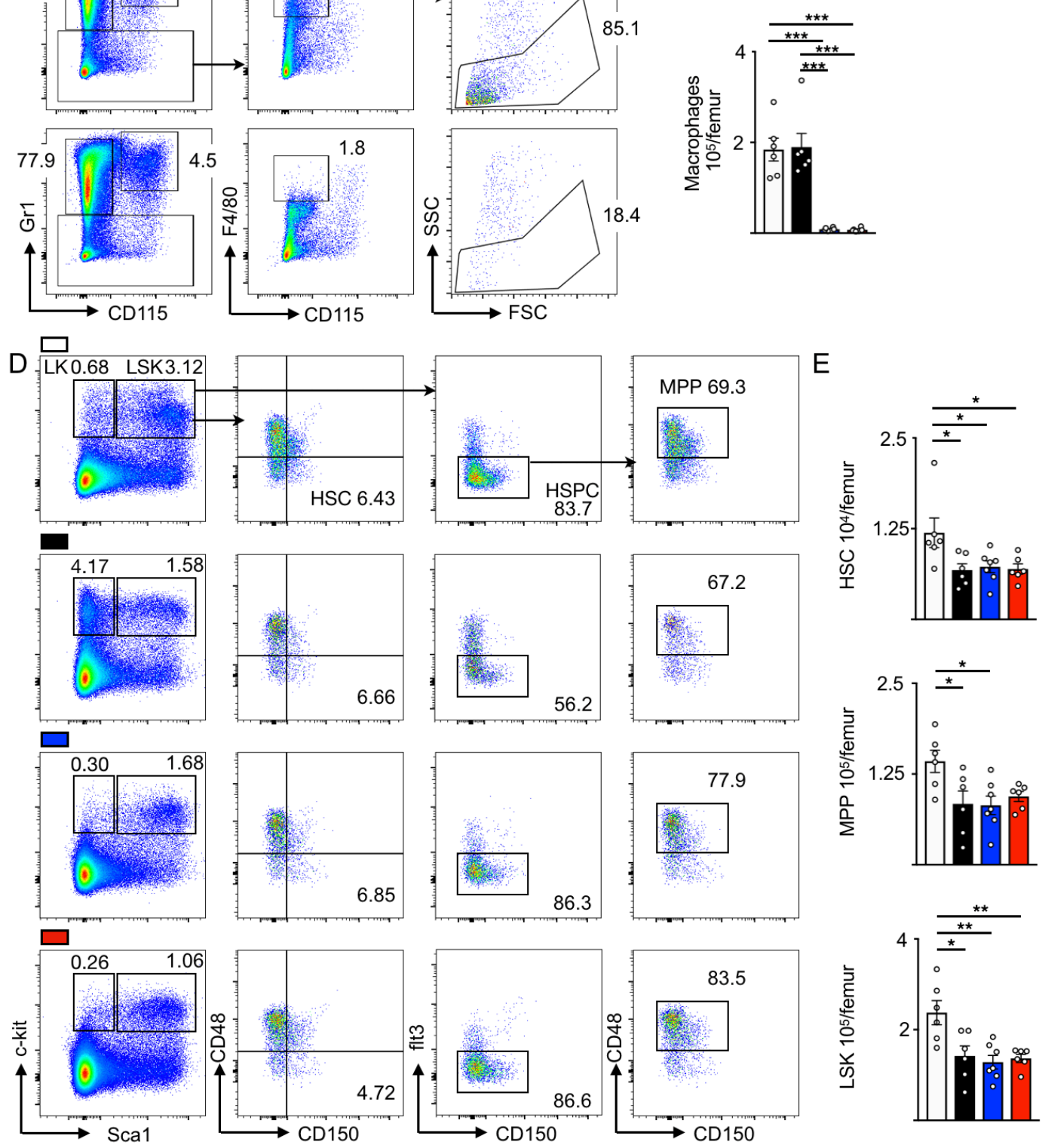

Figure 7. Macrophage ablation abolishes bone marrow memory. (A) Experimental design. Mice received either control liposomes or clodronate liposomes. Half of the mice in each cohort underwent LAD MI. All mice were injected with LPS. (B) Dot plots and (C) quantification of bone marrow macrophages showing efficient depletion by clodronate liposomes. (D) FACS-plots and (E) cell numbers of bone marrow HSPC after clodronate depletion. ${ }^{*} p<0.05,{ }^{* *} p<0.01,{ }^{* * *} p<0.001, n=5-8$, one-way analysis of variance (ANOVA) followed by Tukey's multiple comparisons test. Data are mean \pm s.e.m.. 


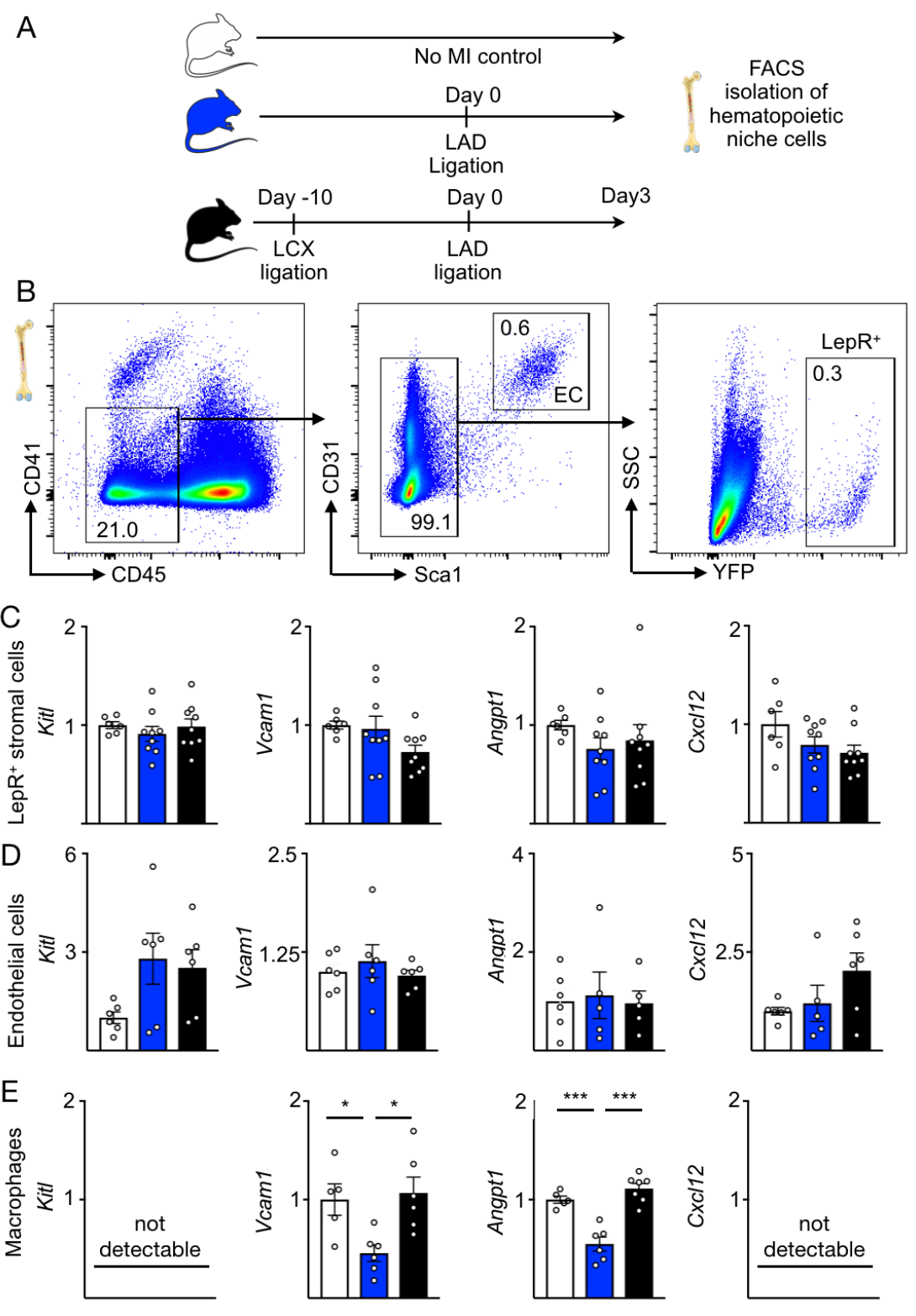

Figure 8. Macrophages attenuate myelopoiesis in recurrent Ml by higher expression of quiescence- and retention-promoting factors. (A) Experimental design. Hematopoietic niche cells were isolated from bone marrow of naive mice and mice with either first or recurrent $\mathrm{MI}$ and subjected to gene expression analysis with qPCR. (B) Gating strategy for isolating bone marrow endothelial cells and LepR ${ }^{+}$stromal cells. (C-E) Gene expression by qPCR in LepR+ stromal cells, endothelial cells and bone marrow macrophages. mRNA levels were normalized to gapdh Ct values. ${ }^{*} p<0.05,{ }^{* *} p<0.01,{ }^{* *} p<0.001, n=5-9$, one-way analysis of variance (ANOVA) followed by Tukey's multiple comparisons test. Data are mean \pm s.e.m. 
A

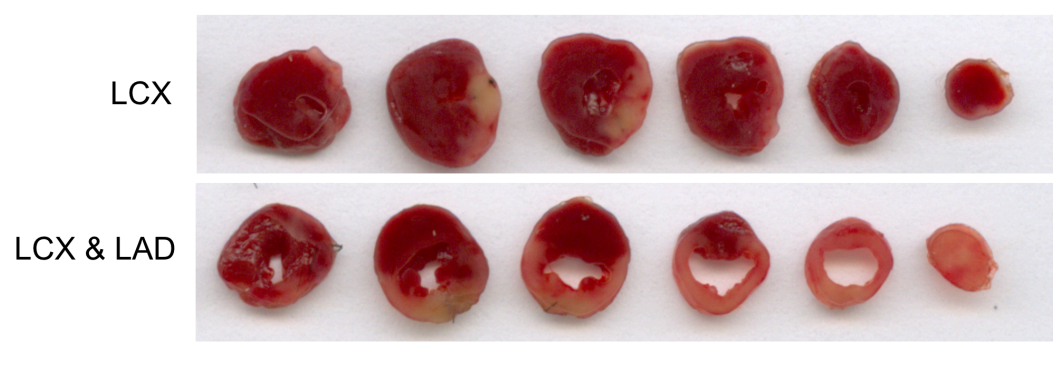

B

$B \square$ LAD ligation

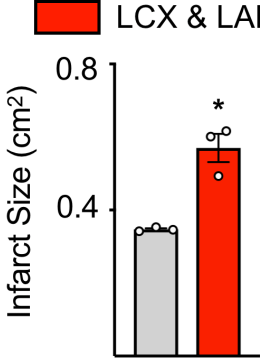

Supplementary Figure 1. 2,3,5-Triphenyltetrazolium chloride (TTC) staining. (A) TTC staining of a heart with an isolated LCX ligation and both ligations. (B) Quantification of infarct size assessed by TTC staining of LAD MIs compared to hearts in which both vessels were ligated. ${ }^{*} p<0.05, n=3$, Student's t-test. Data are mean \pm s.e.m.. 
A

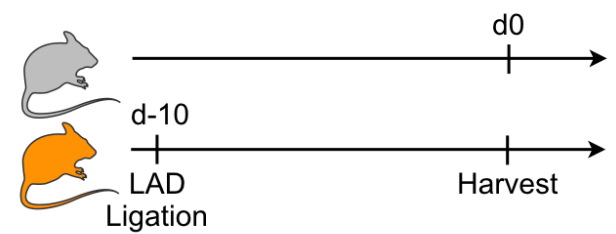

B
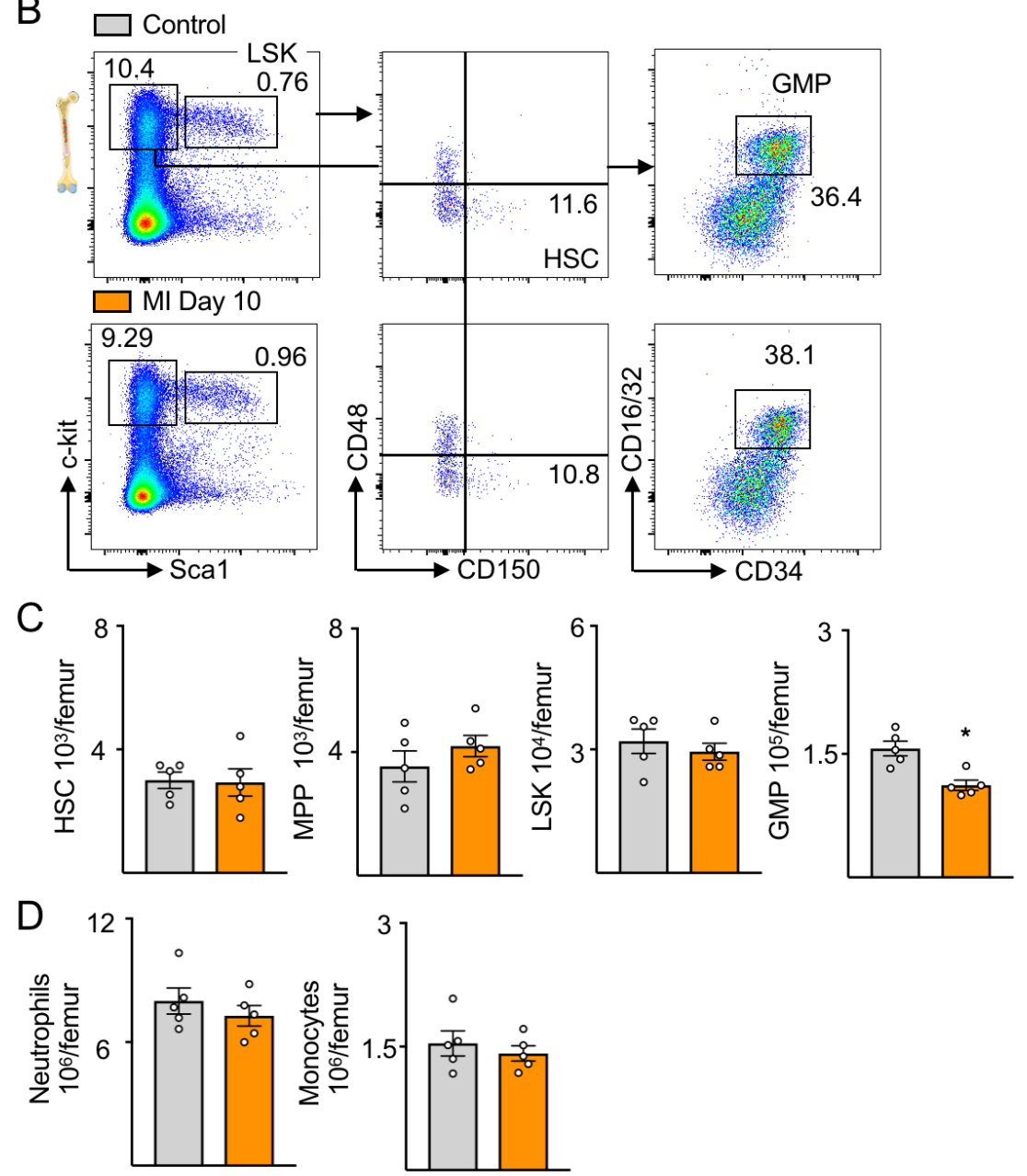

Supplementary Figure 2. HSPC numbers 10 days after MI. (A) Experimental outline. Mice had LAD MI 10 days before bone marrow analysis. (B) Dot plots and (C) quantification of HSPC in bone marrow of mice with MI compared to naive mice. (D) Numbers of mature leukocytes in bone marrow. ${ }^{*} p<0.05, n=5$ Student's t-test. Data are mean \pm s.e.m.. 


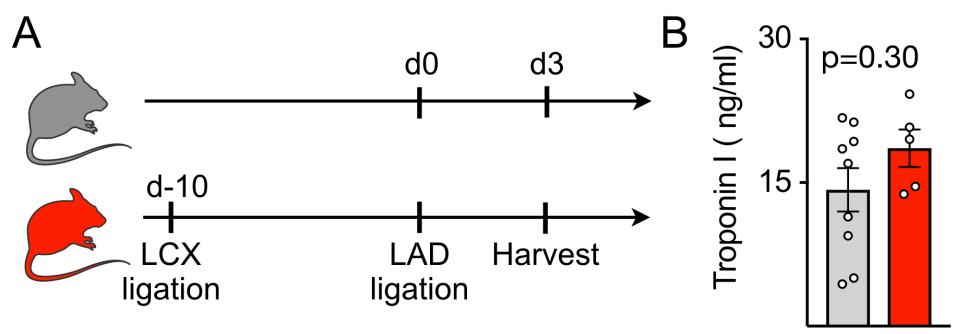

Supplementary Figure 3. Troponin after a first versus recurrent MI. (A) Experimental design. (B) Troponin levels after LAD ligation as the first compared to recurrent MI. $n=5-9$. 
A

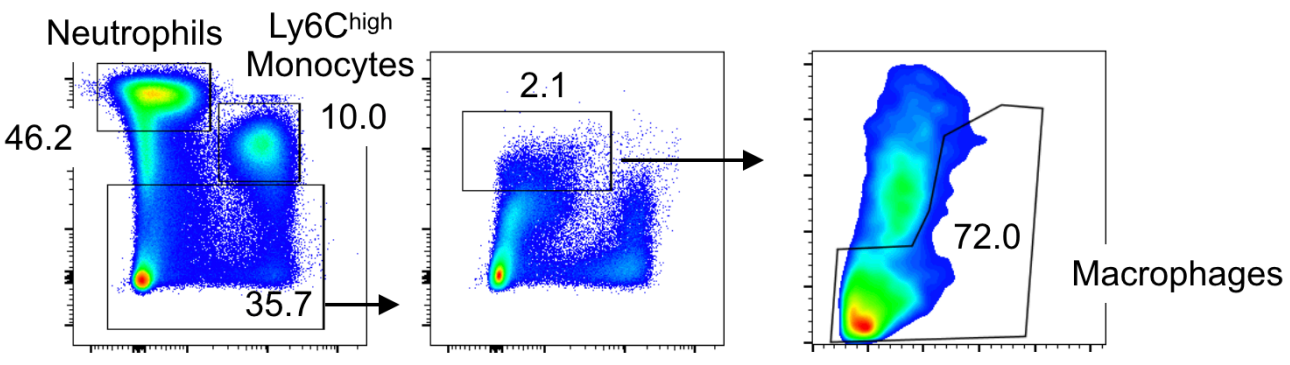

B
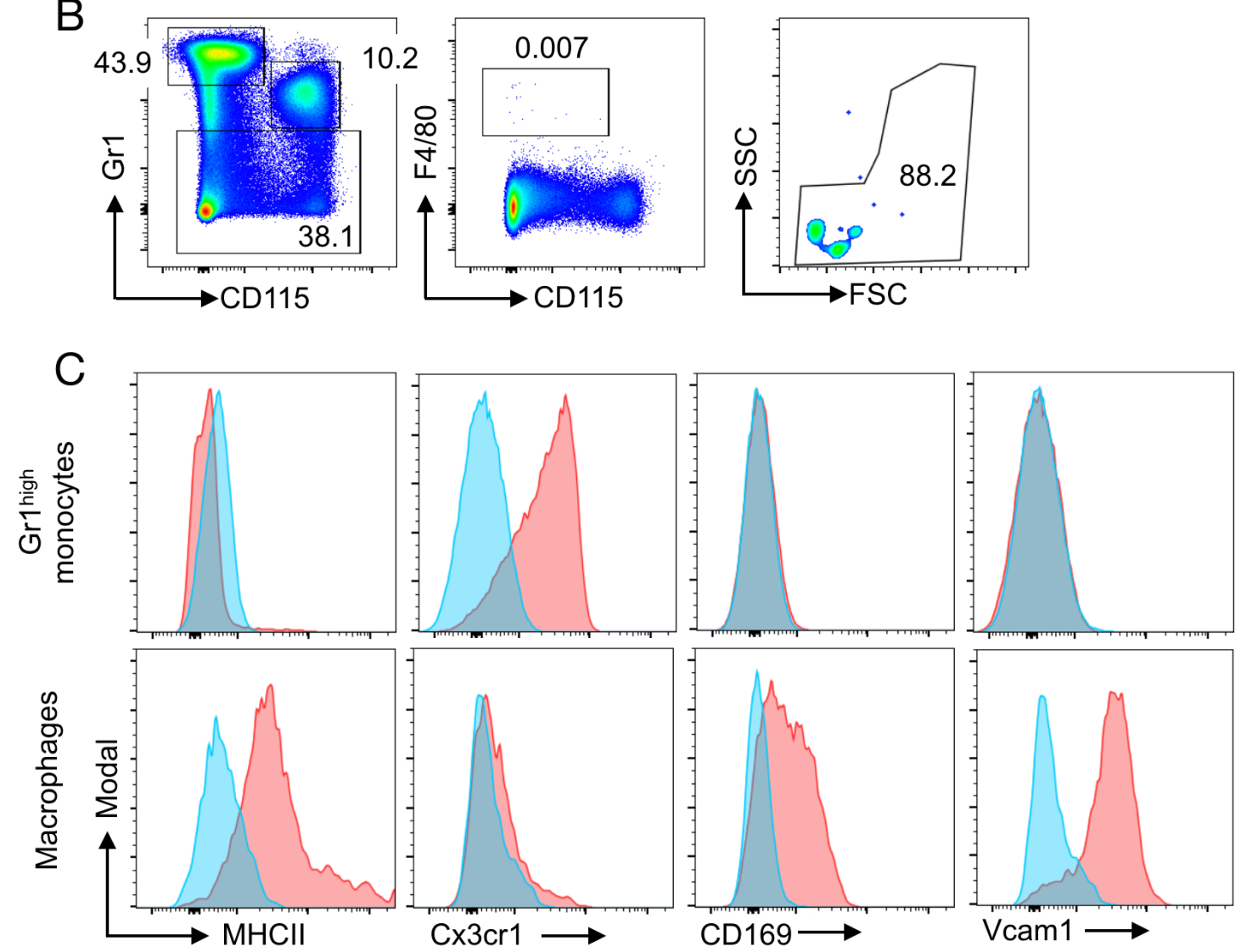

Supplementary Figure 4. Macrophage gating. (A) Gating strategy for bone marrow macrophages with antibody directed against F4/80 and (B) isotype control. (C) Histograms in red depicting the expression of MHCII, Cx3cr1, CD169 and Vcam1 in Gr1hi monocytes and bone marrow macrophages. Isotype controls are shown in blue. 
Supplementary Video 1: MRI short axis cine loop of LCX infarct. Delayed enhancement MRI short axis views of a mouse with LCX MI demonstrating injury of posterolateral left ventricular wall.

Supplementary Video 2: MRI short axis cine loop after recurrent MI (LCX followed by LAD ligation). Delayed enhancement MRI short axis views of the same mouse as in supplementary video 1 , now also showing injury of the anterior left ventricular wall. 